\title{
Epistemic considerations of decision making in games
}

\author{
Mamoru Kaneko* \\ Institute of Policy and Planning Sciences, University of Tsukuba, Ibaraki 305, Japan
}

\begin{abstract}
From the Ex Ante point of view, an axiomatization of decision making in a game with pure strategies is given, while considering its epistemic aspects in propositional game (epistemic) logic. Our axiomatization consists of four base axioms for predicted final decisions. One of them is an epistemic requirement, which together with the others leads to an infinite regress of the knowledge of these axioms. The resulting outcome of this regress is expressed as the common knowledge of the base axioms. We give meta-theoretical evaluations of the derivation of this infinite regress, and consider its implications in solvable and unsolvable games. For a solvable game, it determines predicted decisions to be the common knowledge of a Nash equilibrium, and for an unsolvable game, it is the common knowledge of a subsolution in Nash's sense. The latter result needs the common knowledge of the additional information of which subsolution would be played. We give also meta-theoretical evaluations of these results. (C) 1999 Elsevier Science B.V. All rights reserved.
\end{abstract}

Keywords: Game Logic; Infinite regress of knowledge; Common knowledge; Final decisions; Nash equilibrium; Solvable and unsolvable games

\section{Game logic approach and meta-theoretic evaluations of some game theoretic considerations}

In this paper, decision making in a game is considered from the Ex Ante point of view in an axiomatic manner. For such decision making, players' knowledge and thinking on the game situation are essential. To describe these epistemic aspects as well as the game situation, we will use the propositional fragment of game logic developed in KanekoNagashima (1996) and (1997a). In the framework of game logic, Kaneko-Nagashima (1991, 1996) presented base axioms for decision making, and showed that these base axioms lead to an infinite regress of the knowledge of the axioms themselves, the result

\footnotetext{
*Corresponding author.

E-mail address: kaneko@shako.sk.tsukuba.ac.jp (M. Kaneko)
} 
of which is expressed as the common knowledge of the axioms. Then they solved this infinite regress in the sense that the common knowledge of the axioms determines the final decisions to be the common knowledge of a Nash equilibrium under the common knowledge assumption of interchangeability of Nash equilibria. The objectives of this paper are to evaluate the derivation of the infinite regress in a meta-theoretic manner, which means to consider whether or not a player can prove certain propositions related to the derivation. We also give a full consideration of decision making in an unsolvable game as well as meta-theoretic evaluations of it.

In Ex Ante decision making, since each player makes a strategy choice before the actual play of a game, the knowledge of the structure of the game as well as predictions on the other players' strategy choices may be needed. In the literature of game theory, the Bayesian approach to this problem has been dominant. In the Bayesian approach, the players' knowledge is described by means of a subjective probability on possible types of each player, and classical game theory is treated as a trivial case - games with complete information. A game with complete information itself is, however, not trivial in that it has at least the description of the constituents of a game. Although the Bayesian approach has been shown to be quite rich in capturing various economic problems, it is incapable of treating players' logical and mathematical abilities as well as their knowledge of the descriptions of a game in a direct manner. This leads us to the development of the game logic framework.

Kaneko-Nagashima (1996) and (1997a) developed a hierarchy of logics, $\mathrm{GL}_{0}, \mathrm{GL}_{1}, \ldots$, $\mathrm{GL}_{m}, \ldots$; and $\mathrm{GL}_{\omega}$, where the nesting depths of the knowledge of players' logical and introspective abilities are bounded by $m$ in $\mathrm{GL}_{m}$, and they are unbounded in $\mathrm{GL}_{\omega}$. They are predicate logics for the purpose of describing real number theory in its scope, since classical game theory often relies upon real number theory. They are also infinitary to discuss common knowledge, but the limit logic $\mathrm{GL}_{\omega}$ is required for the full discussion of the problem of common knowledge. The objectives of Kaneko-Nagashima, (1996) and (1997a) were to develop the new framework and to show some possible applications. The purpose of this paper is to give fuller discussions on the epistemic axiomatization of the Ex Ante decision making in a game. For this purpose, we confine ourselves to finite games with pure strategies, for which the propositional fragment of $\mathrm{GL}_{\omega}$ suffices, but any $\mathrm{GL}_{m}(m<\omega)$ is insufficient for our arguments.

Now we describe our game theoretical problems. We meet two kinds of basic problems arising in the Ex Ante decision making:

(i): solution-theoretic problems;

(ii): existence-playability problems.

The first is the problem of how strategy choices are made; and the other is the existence and playability of the solution concept obtained in (i). These problems interact with each other. In this paper, we give the solution-theoretic consideration of the Ex Ante decision making problem for a finite game with pure strategies, and will give also some results on the playability problem.

When we restrict our attention to games with pure strategies, some games might not be playable in the sense that there are no Nash equilibria in pure strategies. When we 
allow mixed strategies, existence is obtained, but some form of real number theory is involved and is assumed to be known to players, which is a stringent requirement. If a game has a Nash equilibrium in pure strategies, playability is not so serious as in the case with mixed strategies, though some playability problems may remain for such a game. From the viewpoint of playability, the cases with and without mixed strategies are totally different. From the solution-theoretic point of view, however, they do not make much differences. The solution-theoretic considerations given in this paper can be carried over to the case with mixed strategies. The existence-playability of a game with mixed strategies is discussed fully in Kaneko (1997a). In this paper, we restrict our attention to games with pure strategies, which enables us to use the propositional fragment of game logic.

A merit of the game logic approach is not only to describe the epistemic aspects of decision making in an explicit manner, but also to enables us to evaluate such descriptions and resulting outcomes from them in a meta-theoretic manner, that is, we can evaluate what the players can or cannot prove. The undecidability result given in Kaneko-Nagashima (1996), that the existence of a unique Nash equilibrium is common knowledge (in mixed strategies) but the players cannot know what the Nash equilibrium is specifically, is an example of such meta-theoretical evaluations. The main contributions of this paper are meta-theoretical evaluations of the axiomatization and of the resulting outcomes from it.

Our axiomatization consists of four base axioms for predicted final decisions:

D1: Best Response to Predicted Decisions - each player's decision is a best response to his predictions on the other players' decisions;

D2: Identical Predictions - each has identical predictions;

D3: Knowledge of Predictions - each knows his own decision and predictions;

D4: Interchangeability of Predicted Decisions - each treats the players as independent decision makers.

The first two axioms simply induce Nash equilibrium, but they together with the third and fourth axioms go much further. The third is an epistemic requirement, and the fourth is a requirement of independent decision making. These two additional requirements differentiate substantially our theory from classical Nash equilibrium theory.

The third axiom together with the second leads to an infinite regress of the knowledge of these four axioms, the result of which is expressed as the common knowledge of those axioms. We will evaluate this derivation in a meta-theoretical manner. The evaluation states that to complete the axiomatization, we meet necessarily the infinite regress. The introduction of an epistemic structure also enables us to demarcate playability from the mere knowledge of the existence of a Nash equilibrium, which will be discussed in Subsection 5.2. One result differentiates the case where the players have abstract knowledge of a game from the case where they have the concrete knowledge of it.

The fourth axiom demarcates between the solvable and unsolvable games in Nash's (1951) sense. For a solvable game, a predicted decision profile is determined to be the common knowledge of a Nash equilibrium. For an unsolvable game, it becomes the 
common knowledge of a subsolution, which is defined also by Nash himself. In this case, the players need to share some information of which subsolution would or not be played. We will prove that without such additional information, the game is not playable. Here we emphasize that although Nash equilibrium plays a central role in our axiomatic consideration, our concern is individual decision-making in a game situation but not an axiomatization of Nash equilibrium. From this point of view, the fourth axiom is indispensable and plays a crucial role in our considerations. In Section 7, the role of each axiom in our axiomatization is considered.

Our axiomatization looks related to Johansen's (1982) informal argument on Nash equilibrium. The seemingly self-referential nature of his argument may be regarded as corresponding to our infinite regress of the base axioms. His claim that his postulates determine a Nash equilibrium for a game with a unique Nash equilibrium is consistent with our results. In this paper, however, we will consider epistemic aspects explicitly and also treat unsolvable games. The comparison between his and ours will be given in Subsection 7.2. This paper is related also to Aumann-Brandenberger (1995) in its objectives. These authors considered some epistemic conditions for Nash equilibrium in a Bayesian framework. The difference is that they concern necessary conditions for Nash equilibrium, while our concern is the complete characterization of predicted final decisions with meta-theoretical evaluations (the direct comparisons are difficult since the frameworks are different).

As already stated, the logic $\mathrm{GL}_{\omega}$ we use is a propositional fragment of game logic of Kaneko-Nagashima (1996) and (1997a). In fact, it is shown in Kaneko (1996) that common knowledge logic developed in Halpern-Moses (1992) and Lismont-Mongin (1994) can be faithfully embedded into our logic (with a slight restriction on our logic). This implies that the results obtained in this paper are all translated into common knowledge logic.

A final remark on $\mathrm{GL}_{\omega}$ is that we assume the Veridicality Axiom $\mathrm{T}_{i}: K_{i}(A) \supset A$, while Axiom $\mathrm{D}_{i}: \neg K_{i}(\neg A \wedge A)$ is adopted in Kaneko-Nagashima, (1996) and (1997a). Thus, we adopt here the S4-type game logic for our presentational purposes, instead of the KD4-type game logic of Kaneko-Nagashima, (1996) and (1997a). However, since the S4-type logic can be faithfully embedded into the KD4-type game logic, the whole argument of this paper can be done in the KD4-type. Finally, we remark that we do not need Axiom $5_{i}: \neg K_{i}(A) \supset K_{i}\left(\neg K_{i}(A)\right)$, called the Negative Introspection, which is often an obstacle for proof-theoretic arguments.

We will repeat some results given in Kaneko-Nagashima (1991) and (1996), but give proofs to some of them for completeness. We distinguish the results already given by putting * from new ones.

\footnotetext{
${ }^{1}$ Bacharach (1987) is the seminal paper along the line of the research of this paper. Nevertheless, his framework is not sufficient to facilitate the considerations of the epistemic aspects - for example, common knowledge is not formulated in his framework explicitly.
} 


\section{Preliminaries}

In Subsection 2.1 we give basic game theoretical concepts in the nonformalized language, and in Subsection 2.2, we give formalized language $\mathscr{P}^{\omega}$ and game logic GL ${ }_{\omega}$. In Subsection 2.3, we redescribe game theoretical concepts in the formalized language $\mathscr{P}^{\omega}$.

\subsection{Game theoretical concepts in the nonformalized language}

Consider an $n$-person finite noncooperative game $g$ in strategic form. The players are denoted by $1, \ldots, n$, and each player $i$ has $\ell_{i}$ pure strategies with $\left(\ell_{i} \geq 2\right)$. We assume that the players do not play mixed strategies. Player $i$ 's strategy space is denoted by $\Sigma_{i}$ := $\left\{\mathbf{s}_{i 1}, \ldots, \mathbf{s}_{i \ell_{i}}\right\}$, and his payoff function is a real-valued function $g_{i}$ on $\Sigma:=\Sigma_{1} \times \cdots \times \Sigma_{n}$ for $i=1, \ldots, n$. We call an element in $\Sigma$ a strategy profile.

A strategy profile $a=\left(a_{1}, \ldots, a_{n}\right)$ is called a Nash equilibrium iff for $i=1, \ldots, n$, $g_{i}(a) \geq g_{i}\left(b_{i} ; a_{-i}\right)$ for all $b_{i} \in \Sigma_{\mathrm{i}}$, where $a_{-i}=\left(a_{1}, \ldots, a_{i-1}, a_{i+1}, \ldots, a_{n}\right)$ and $\left(b_{i} ; a_{-i}\right)=$ $\left(a_{1}, \ldots, a_{i-1}, b_{i}, a_{i+1}, \ldots, a_{n}\right)$. We denote the set of Nash equilibria of game $g$ by $E_{g}$.

Consider a maximal nonempty subset $E$ of $E_{g}$ which satisfies the interchangeability condition:

$$
a, b \in E \text { and } i=1, \ldots, n \text { imply }\left(a_{i} ; b_{-i}\right) \in E .
$$

This is equivalent to that $a^{1}, \ldots, a^{n} \in E$ implies $\left(a_{1}^{1}, \ldots, a_{n}^{n}\right) \in E$, which states that if each player $i$ independently chooses his equilibrium strategy $a_{i}^{i}$, the resulting profile $\left(a_{1}^{1}, \ldots, a_{n}^{n}\right)$ is also an equilibrium. We call such a maximal set a subsolution, which was introduced by Nash (1951). Each Nash equilibrium belongs to at least one subsolution. We denote the subsolutions of game $g$ by $E_{g}^{1}, \ldots, E_{g}^{\sigma}$. We stipulate that when $E_{g}$ is empty, $\sigma=0$. When $E_{g}$ is nonempty, $\sigma \geq 1$. When $\sigma=1$, game $g$ is said to be solvable.

The game (Battle of the Sexes) of Table 1 has $E_{g}=E_{g}^{1} \cup E_{g}^{2}=\left\{\left(\mathbf{s}_{11}, \mathbf{s}_{21}\right)\right\} \cup$ $\left\{\left(\mathbf{s}_{12}, \mathbf{s}_{22}\right)\right\}$, and is not solvable. The game of Table 2 is not solvable either and has $E_{g}=E_{g}^{1} \cup E_{g}^{2}=\left\{\left(\mathbf{s}_{11}, \mathbf{s}_{21}\right),\left(\mathbf{s}_{11}, \mathbf{s}_{22}\right)\right\} \cup\left\{\left(\mathbf{s}_{11}, \mathbf{s}_{21}\right),\left(\mathbf{s}_{12}, \mathbf{s}_{21}\right)\right\}$. Here $\left(\mathbf{s}_{11}, \mathbf{s}_{21}\right)$ belongs to both

Table 1

\begin{tabular}{lll}
\hline & $\mathbf{s}_{21}$ & $\mathbf{s}_{22}$ \\
\hline $\mathbf{s}_{11}$ & $(2,1)^{*}$ & $(0,0)$ \\
$\mathbf{s}_{12}$ & $(0,0)$ & $(1,2)^{*}$ \\
\hline
\end{tabular}

\footnotetext{
${ }^{2}$ Zero-sum two-person games are solvable if they have Nash equilibria. Other sufficient conditions for solvability are found in Kats-Thisse (1992). A study of subsolutions is found in Jansen (1981) (see also its references).
} 
Table 2

\begin{tabular}{lll}
\hline & $\mathbf{s}_{21}$ & $\mathbf{s}_{22}$ \\
\hline $\mathbf{s}_{11}$ & $(2,2)^{*}$ & $(1,2)^{*}$ \\
$\mathbf{s}_{12}$ & $(2,1)^{*}$ & $(0,0)$ \\
\hline
\end{tabular}

subsolutions. The games of Tables 3 (Prisoner's Dilemma) and 4 have the same equilibrium sets $E_{g}=\left\{\left(\mathbf{s}_{12}, \mathbf{s}_{22}\right)\right\}$ and are solvable.

In the next subsection, we give a formal logic in which a game as well as such other epistemic constituents are described.

\subsection{Game logic $G L_{\omega}$}

In the following, we use some notions of predicate logic. Since, however, we use neither variables nor quantifiers, the following logic is essentially propositional.

We start with the following list of symbols:

constant symbols: $\mathbf{s}_{11}, \ldots, \mathbf{s}_{1 \ell_{1}} ; \mathbf{s}_{21}, \ldots, \mathbf{s}_{2 \ell_{2}} ; \ldots ; \mathbf{s}_{n 1}, \ldots, \mathbf{s}_{n \ell_{n}} ;$

binary predicate symbols: $=$;

$2 n$-ary predicate symbols: $R_{1}, \ldots, R_{n}$;

n-ary predicate symbols: $D_{1}, \ldots, D_{n}$;

knowledge operator symbols: $K_{1}, \ldots, K_{n}$;

logical connectives: $\neg$ (not), $\supset$ (implies), $\wedge$ (and), $\vee$ (or) ;

parentheses: ( , ).

As in Subsection 2.1, the constants $\mathbf{s}_{11}, \ldots, \mathbf{s}_{1 \ell_{1}} ; \mathbf{s}_{21}, \ldots, \mathbf{s}_{2 \ell_{2}} ; \ldots ; \mathbf{s}_{n 1}, \ldots, \mathbf{s}_{n \ell_{n}}$ are the players' strategies. The binary predicate symbol $=$ is intended to describe identity between strategies for each single player. The $2 n$-ary symbol $R_{i}(\cdot: \cdot)$ is used to describe player $i$ 's payoff function $g_{i}$. The $n$-ary symbol $D_{i}(\cdot)$ is to describe $i$ 's prediction of the players' strategy choices, that is, $D_{i}\left(a_{1}, \ldots, a_{n}\right)$ means that $i$ predicts that $1, \ldots, n$ could choose $a_{1}, \ldots, a_{n}$ as final decisions in their ex ante decision making. Of course, $a_{i}$ itself is $i$ 's own possible decision. These $D_{1}, \ldots, D_{n}$ will be determined by the nonlogical axioms which will be given in Section 3. By the expression $K_{i}(A)$, we mean that player $i$ knows a formula $A$.

Table 3

\begin{tabular}{lll}
\hline & $\mathbf{s}_{21}$ & $\mathbf{s}_{22}$ \\
\hline $\mathbf{s}_{11}$ & $(5,5)$ & $(1,6)$ \\
$\mathbf{s}_{12}$ & $(6,1)$ & $(3,3)^{*}$ \\
\hline
\end{tabular}

Table 4

\begin{tabular}{lll}
\hline & $\mathbf{s}_{21}$ & $\mathbf{s}_{22}$ \\
\hline $\mathbf{s}_{11}$ & $(5,5)$ & $(1,2)$ \\
$\mathbf{s}_{12}$ & $(6,1)$ & $(3,3)^{*}$ \\
\hline
\end{tabular}


First, we develop the space of formulae. For any strategies $a_{j}, b_{j}$ in $\Sigma_{j}\left(a_{j}\right.$ and $b_{j}$ may be identical and $j=1, \ldots, n),\left(a_{j}=b_{j}\right)$ is an atomic formula, and for strategy profiles $\left(a_{1}, \ldots, a_{n}\right),\left(b_{1}, \ldots, b_{n}\right)$ in $\Sigma$, the expressions $R_{i}\left(a_{1}, \ldots, a_{n}: b_{1}, \ldots, b_{n}\right)$ and $D_{i}\left(a_{1}, \ldots, a_{n}\right) \quad(i=$ $1, \ldots, n)$ are also atomic formulae. These atomic formulae correspond to propositional variables in the standard formulation of propositional logic. Since the number of strategies is finite, so is the number of atomic formulae.

Let $\mathscr{P}^{0}$ be the set of all formulae generated by the standard finitary inductive definition with respect to $\neg, \supset$ and $K_{1}, \ldots, K_{n}$ from the atomic formulae. That is, $\mathscr{P}^{0}$ is the set of 0-formulae defined by the following induction:

$(\mathbf{0}-\mathbf{i})$ : any atomic formula is a 0 -formula;

(0-ii): if $A$ and $B$ are 0 -formulae, so are $(\neg A),(A \supset B)$ and $K_{i}(A)^{3}{ }^{3}$

Suppose that the set $\mathscr{P}^{k-1}$ of $(k-1)$-formulae is already defined $(k=1, \ldots)$. Then $\mathscr{P}^{k}$ is the set of $k$-formulae defined by the following induction:

(k-i): any expression in $\mathscr{P}^{k-1} \cup\{(\wedge \Phi),(\vee \Phi): \Phi$ is a nonempty countable subset of $\left.\mathscr{P}^{k-1}\right\}$ is a $k$-formula;

(k-ii): if $A$ and $B$ are $k$-formulae, so are $(\neg A),(A \supset B)$ and $K_{i}(A)$.

We denote $\bigcup_{k<\omega} \mathscr{P}^{k}$ by $\mathscr{P}^{\omega}$. An expression in $\mathscr{P}^{\omega}$ is called simply a formula. We abbreviate $\wedge\{A, B\}$ and $\vee\{A, B\}$ as $A \wedge B$ and $A \vee B$, and $(A \supset B) \wedge(B \supset A)$ as $A \equiv B$. We also abbreviate some parentheses in the standard manner. Also, we call $\Phi$ an allowable set iff $\Phi$ is a nonempty countable subset of $\mathscr{P}^{k}$ for some $k<\omega$. We say that a formula $A$ is nonepistemic iff it contains no $K_{i}, i=1, \ldots, n$.

The primary reason for our infinitary language is to express common knowledge explicitly as a conjunctive formula. The common knowledge of a formula $A$ is defined as follows: For any $m \geq 0$, we denote the set $\left\{K_{i_{1}} K_{i_{2}} \ldots K_{i_{m}}\right.$ : each $K_{i_{t}}$ is one of $K_{1}, \ldots, K_{n}$ and $i_{t} \neq i_{t+1}$ for $\left.t=1, \ldots, m-1\right\}$ by $K(m)$. We assume that $K(0)$ consists of the null symbol $e$ (i.e., $e(A)$ is $A$ itself for any $A$ ). We define the common knowledge formula of $A$ as

$$
\wedge\left\{K(A): K \in \bigcup_{m<\omega} K(m)\right\},
$$

which we denote by $C(A)$. If $A$ is in $\mathscr{P}^{k-1}$, the set $\left\{K(A): K \in \bigcup_{m<\omega} K(m)\right\}$ is a countable subset of $\mathscr{P}^{k-1}$, and its conjunction, $C(A)$, belongs to $\mathscr{P}^{k}$ by $(\mathrm{k}-\mathrm{i})$. Hence the space $\mathscr{P}^{\omega}$ is closed with respect to the operation $C(\cdot)$.

Note that $A$ itself is included as a conjunct in $C(A)$, since $K(0)=\{e\}$. In this sense, $C(A)$ is "common knowledge" instead of "common belief" which is defined to be the conjunction obtained from (2.2) by excluding $A$.

Base logic $\mathrm{GL}_{0}$ is defined by the following five axiom schemata and three inference rules: for any formulae $A, B, C$ and allowable set $\Phi$,

(L1): $A \supset(B \supset A)$;

(L2): $\quad(A \supset(B \supset C)) \supset((A \supset B) \supset(A \supset C))$;

(L3): $\quad(\neg A \supset \neg B) \supset((\neg A \supset B) \supset A)$;

(L4): $\wedge \Phi \supset A$, where $A \in \Phi$;

${ }^{3}$ and all 0 -formulae are obtained by a finite applications of those steps. We will not add this qualification in the following inductive definitions. 
(L5): $\quad A \supset \vee \Phi$, where $A \in \Phi$;

$$
\begin{aligned}
& \frac{A \supset B \quad A}{B}(M P) \\
& \frac{\{A \supset B: B \in \Phi\}}{A \supset \wedge \Phi}(\wedge-\text { Rule }) \\
& \frac{\{A \supset B: A \in \Phi\}}{\vee \Phi \supset B}(\vee-\text { Rule }) .
\end{aligned}
$$

These axioms and inference rules determine base logic $\mathrm{GL}_{0}$.

We define game logic $\mathrm{GL}_{\omega}$ by adding the following axiom schemata and inference rule to $\mathrm{GL}_{0}$ : for any formulae $A, B$ and $i=1, \ldots, n$;

$\left(\mathrm{MP}_{i}\right): K_{i}(A \supset B) \wedge K_{i}(A) \supset K_{i}(B)$;

$\left(\mathrm{T}_{i}\right): \quad K_{i}(A) \supset A$;

$\left(\mathrm{PI}_{i}\right): K_{i}(A) \supset K_{i} K_{i}(A)$;

(C-Barcan): $\wedge\left\{K_{i} K(A): K \in \bigcup_{m<\omega} K(m)\right\} \supset K_{i} C(A)$;

and

(Necessitation): $\frac{A}{K_{i}(A)}$.

We will abbreviate Necessitation as $\mathrm{Nec}$, and use $\mathrm{MP}_{i}, \mathrm{~T}_{i}, \mathrm{PI}_{i}$ as generic names for those for different $i$.

A proof $P$ in $\mathrm{GL}_{\omega}$ is a countable tree with the following properties: (i) every path from the root is finite; (ii) a formula is associated with each node, and the formula associated with each leaf is an instance of the axioms; and (iii) adjoining nodes together with their associated formulae form an instance of the above inferences. We write $\vdash_{\omega} A$ iff there is a proof $P$ such that $A$ is associated with the root. For any subset $\Gamma$ of $\mathscr{P}^{\omega}$, we write $\Gamma \vdash_{\omega} A$ iff $\vdash_{\omega} \wedge \Phi \supset A$ for some nonempty finite subset $\Phi$ of $\Gamma$. When $\Gamma$ is empty, $\Gamma \vdash{ }_{\omega} A$ is assumed to be $\vdash_{\omega} A$ itself. We also abbreviate $\Gamma \cup \Theta \vdash_{\omega} A$ and $\Gamma \cup\{B\} \vdash{ }_{\omega} A$ as $\Gamma, \Theta \vdash_{\omega} A$ and $\Gamma, B \vdash_{\omega} A$, etc.

When we restrict the use of axioms and inference rules to those of $\mathrm{GL}_{0}$, we denote the provability relation by $\vdash_{0}$. Logic $\mathrm{GL}_{0}$ is an infinitary extension of classical finitary propositional logic.

We will use the following facts without references (see Kaneko-Nagashima (1996)).

Lemma 2.1. Let $\Gamma, \Theta$ be sets of formulae, and $\Phi$ an allowable set of formulae. Then

1. $\vdash_{\omega}(A \wedge B \supset C) \equiv(A \supset(B \supset C))$;

2. if $\Gamma \vdash{ }_{\omega} A \supset B$ and $\Theta \vdash{ }_{\omega} B \supset C$, then $\Gamma, \Theta \vdash{ }_{\omega} A \supset C$;

3. $\vdash_{\omega} \wedge \Phi$ if and only if $\vdash_{\omega} A$ for all $A \in \Phi$;

4. $\vdash_{\omega} K_{i}(\wedge \Phi) \supset \wedge K_{i}(\Phi)$, and if $\Phi$ is a finite set, then $\vdash_{\omega} K_{i}(\wedge \Phi) \equiv \wedge K_{i}(\Phi)$, where $K_{i}(\Phi)$ is the set $\left\{K_{i}(A): A \in \Phi\right\}$

\footnotetext{
${ }^{4}$ Since $\mathrm{GL}_{\omega}$ has Nec, nonlogical axioms should be introduced in this manner, instead of being initial formulae in a proof. For the treatment of nonlogical axioms in a logic with Nec, see Kaneko-Nagashima (1997b).
} 
5. $\vdash_{\omega} \vee K_{i}(\Phi) \supset K_{i}(\vee \Phi)$.

Note that (1)-(3) hold also for $\vdash_{0}$.

Axiom $\mathrm{MP}_{i}$ and inference rule $\mathrm{Nec}$ in addition to $\mathrm{GL}_{0}$ give the complete logical ability to each player (see (Kaneko and Nagashima, 1996)). Axiom $\mathrm{T}_{i}$, which is called Veridicality Axiom, states that if $i$ knows $A$, then $A$ is true from the objective point of view. Axiom $\mathrm{PI}_{i}$, called the Positive Introspection, means that if player $i$ knows $A$, he knows that he knows $A$. In fact, these logical and introspective abilities of the players are common knowledge in $\mathrm{GL}_{\omega}$ (see (Kaneko and Nagashima, 1996)).

Axiom C-Barcan is called the common knowledge Barcan axiom. For the development of our framework, C-Barcan will be used to derive the property:

$$
\vdash_{\omega} C(A) \supset K_{i} C(A) \text { for } i=1, \ldots, n .
$$

That is, if $A$ is common knowledge, then each player $i$ knows that it is common knowledge. This property will play an important role in the epistemic axiomatization of final decisions in later sections.

Proof of (2.3)*. Let $K$ be an arbitrary element in $\bigcup_{m<\omega} K(m)$. When $K_{i}$ is not the outermost symbol of $K$, we have $\vdash_{\omega} C(A) \supset K_{i} K(A)$ by L4. When $K_{i}$ is the outermost symbol of $K$, we have $\vdash_{\omega} K(A) \supset K_{i} K(A)$ by $\mathrm{PI}_{i}$. This together with $\vdash_{\omega} C(A) \supset K(A)$ implies $\vdash_{\omega} C(A) \supset K_{i} K(A)$. Thus $\vdash_{\omega} C(A) \supset K_{i} K(A)$ for all $K \in \bigcup_{m<\omega} K(m)$. Hence $\vdash_{\omega} C(A) \supset \wedge\left\{K_{i} K(A): \quad K \in \bigcup_{m<\omega} K(m)\right\}$ by $\wedge$-Rule. By C-Barcan, we obtain $\vdash_{\omega} C(A) \supset K_{i} C(A)$.

Since the finitary propositional modal logic defined by $\mathrm{MP}_{i}, \mathrm{~T}_{i}, \mathrm{PI}_{i}$ and $\mathrm{Nec}$ in addition to classical propositional logic is called S4, our logic is an infinitary extension of multi-modal S4 with C-Barcan. Kaneko-Nagashima (1996) and (1997a) assumed the following weaker axiom:

$$
\left(\perp_{i}\right): \neg K_{i}(\neg A \wedge A) \text {; }
$$

instead of Axiom $\mathrm{T}_{i}$. Axiom $\perp_{i}$ is called $\mathrm{D}$ in the literature of modal logic. Hence Kaneko-Nagashima (1996) and (1997a) developed the KD4-type game logic. In the KD4-type game logic, since knowledge is not necessarily objectively true, it is called belief. In fact, knowledge and belief are more fully discussed in the KD4-type game logic. However, we do not treat our subjects in the way that the difference in them is reflected. From the viewpoint of presentational purposes, the S4-type game logic is more convenient than the KD4-type. Hence we adopt the S4-type game logic.

In the subsequent analysis, the relation between the provabilities of $\mathrm{GL}_{\omega}$ and $\mathrm{GL}_{0}$ is important. For this purpose, we introduce the following notion. Let $A$ be a formula in $\mathscr{P}^{\omega}$. Then $\varepsilon A$ is the formula obtained from $A$ by eliminating all the occurrences of $K_{1}, \ldots, K_{n}$ in $A$, and $\varepsilon \Gamma$ is the set $\{\varepsilon A: A \in \Gamma\}$ for any set $\Gamma$ of formulae. Formula $\varepsilon A$ is nonepistemic, and if $A$ is nonepistemic, $\varepsilon A$ is $A$ itself. For example, $\varepsilon\left(K_{i}(A) \wedge K_{i}(A \supset\right.$ $\left.B) \supset K_{i}(B)\right)$ is $\varepsilon A \wedge(\varepsilon A \supset \varepsilon B) \supset \varepsilon B$, and $\left.\varepsilon\left(K_{i}(A) \supset A\right)\right)$ is $(\varepsilon A \supset \varepsilon A)$. For every instance $A$ of the epistemic axioms, $\varepsilon A$ is a nonepistemic provable formula with respect to $\vdash_{0}$ and 
every instance of $\mathrm{Nec}$ becomes a trivial inference with an application of $\varepsilon$. This is the reason for Lemma 2.2.(1). We have also the other lemmas (see Kaneko-Nagashima (1996)).

\section{Lemma 2.2.}

1. If $\Gamma \vdash_{\omega} A$, then $\varepsilon \Gamma \vdash_{0} \varepsilon A$.

2. If $\Gamma \vdash_{0} A$ or $\Gamma \vdash_{\omega} A$, then $C(\Gamma) \vdash_{\omega} C(A)$, where $C(\Gamma)=\{C(B): B \in \Gamma\}$.

\section{Lemma 2.3.}

1. $\vdash_{\omega} C(A \supset B) \supset(C(A) \supset C(B))$;

2. if $C(\Gamma) \vdash_{\omega} A$, then $C(\Gamma) \vdash_{\omega} K_{i}(A)$ for $i=1, \ldots, n$.

Lemma 2.4. Let $\Phi$ be an allowable set of formulae. Then

1. $\vdash_{\omega} C(\wedge \Phi) \supset \wedge C(\Phi)$, and if $\Phi$ is a finite set, then $\vdash_{\omega} C(\wedge \Phi) \equiv \wedge C(\Phi)$;

2. $\vdash_{\omega} \vee C(\Phi) \supset C(\vee \Phi)$.

Although our analysis is primarily syntactical, we will use some semantic methods in several places. For this purpose, we prepare some semantics and review some results to be used for the subsequent analysis. We say that a formula $A$ is finitary iff it contains no infinitary conjunction and no infinitary disjunctions. We denote the set of all nonepistemic finitary formulae by $\mathscr{P}^{\mathrm{f}}$. The space $\mathscr{P}^{\mathrm{f}}$ is closed with respect to $\neg, \supset$ and finitary $\wedge, \vee$. When we restrict the language of base logic $\mathrm{GL}_{0}$ to $\mathscr{P}^{\mathrm{f}}$, the resulting logic is classical propositional logic, which we denote by $\mathrm{GL}_{0}^{\mathrm{f}}$. Base logic $\mathrm{GL}_{0}$ is a conservative extension of $\mathrm{GL}_{0}^{\mathrm{f}}$, i.e., for any $A \in \mathscr{P}^{\mathrm{f}}$,

$$
\text { if } \vdash_{0} A \text {, then } \vdash_{0}^{\mathrm{f}} A \text {, }
$$

where $\vdash_{0}^{\mathrm{f}}$ is the provability relation of $\mathrm{GL}_{0}^{\mathrm{f}}$. Of course, the converse of (2.4) holds.

We will use the classical two-valued semantics for $\mathrm{GL}_{0}^{\mathrm{f}}$. An assignment $\tau$ is a function from the set of atomic formulae to true,false\}. We define the truth relation $\vDash{ }_{\tau}$ relative to an assignment $\tau$ by the following induction on the structure of a formula in $\mathscr{P}^{\mathrm{f}}$ :

(T0): for any atomic formula $A, \vDash{ }_{\tau} A$ iff $\tau(A)=$ true;

(T1): $\vDash{ }_{\tau} \neg A$ iff not $\vDash{ }_{\tau} A$;

(T2): $\vDash{ }_{\tau} A \supset B$ iff not $\vDash{ }_{\tau} A$ or $\vDash{ }_{\tau} B$;

(T3): $\vDash{ }_{\tau} \wedge \Phi$ iff $\vDash{ }_{\tau} A$ for all $A \in \Phi$;

(T4): $\vDash{ }_{\tau} \vee \Phi$ iff $\vDash{ }_{\tau} A$ for some $A \in \Phi$.

The following is the standard soundness-completeness theorem: for any $A \in \mathscr{P}^{\mathrm{f}}$,

$$
\vdash_{0}^{\mathrm{f}} A \text { if and only if } \models{ }_{\tau} A \text { for any assignment } \tau \text {. }
$$

The only-if part is equivalent to that if there is an assignment $\tau$ such that $\vDash{ }_{\tau} A$, then $A$ is consistent with respect to $\vdash_{0}^{\mathrm{f}}$ (a fortiori, with respect to $\vdash_{0}$ by (2.4)). We will refer to this as Soundness for $\mathrm{GL}_{0}^{\mathrm{f}}$. 


\subsection{Game theoretical concepts in the formalized language $\mathscr{P}^{\omega}$}

Now we describe the game theoretical concepts given in Subsection 2.1 in $\mathscr{P}^{\omega}$.

First, we make the following axiom: for all distinct $a_{i}, b_{i} \in \Sigma_{i}(i=1, \ldots, n)$,

Axiom (Eq). $a_{i}=a_{i}$ and $\neg\left(a_{i}=b_{i}\right)$.

We denote the set of instances of this axiom by Eq, which is a finite set of formulae.

Second, we describe the payoff functions $g_{1}, \ldots, g_{n}$ in terms of symbols $R_{1}, \ldots, R_{n}$ as follows: for strategy profiles $a, b, a^{\prime}, b^{\prime}$ with $g_{i}(a) \geq g_{i}(b)$ and $g_{i}\left(a^{\prime}\right)<g_{i}\left(b^{\prime}\right)$ and $i=$ $1, \ldots, n$,

$\operatorname{Axiom}\left(\mathbf{G}_{g}\right) . R_{i}(a ; b)$ and $\neg R_{i}\left(a^{\prime} ; b^{\prime}\right)$.

We denote by $\mathrm{G}_{g}$ the set of all instances of this axiom. This describes the payoff functions $g_{1}, \ldots, g_{n}$ by preferences $R_{1}, \ldots, R_{n}$. It holds that for any $a, b \in \Sigma$, either $\mathrm{G}_{g}$ $\vdash_{0} R_{i}(a ; b)$ or $\mathrm{G}_{g} \vdash_{0} \neg R_{i}(a ; b)$.

In the game of Table 1, Eq is the set $\left\{\mathbf{s}_{i t}=\mathbf{s}_{i t}: i=1,2\right.$ and $\left.t=1,2\right\} \cup\left\{\neg \mathbf{s}_{i t}=\mathbf{s}_{i t^{\prime}}: i=1,2\right.$ and $t, t^{\prime}=1,2$ with $\left.t \neq t^{\prime}\right\}$, and $\mathrm{G}_{g}$ is the set of the preferences of players 1 and 2 . Note that $\mathrm{G}_{g}$ contains both positive and negative preferences.

We define the Nash equilibrium property to be the formula:

$$
\bigwedge_{i=1}^{n} \wedge_{y_{i} \in \Sigma_{i}} R_{i}\left(a: y_{i} ; a_{-i}\right)
$$

which we denote by $\operatorname{Nash}(a)$. Since either $\mathrm{G}_{g} \vdash_{0} R_{i}(a ; b)$ or $\mathrm{G}_{g} \vdash_{0} \neg R_{i}(a ; b)$ for any $a, b \in \Sigma, \operatorname{Nash}(a)$ is also decidable for any $a \in \Sigma$ under $\mathrm{G}_{g}$, that is,

$$
\text { either } \mathrm{G}_{g} \vdash_{0} \operatorname{Nash}(a) \text { or } \mathrm{G}_{g} \vdash_{0} \neg \operatorname{Nash}(a) \text {. }
$$

Also, it holds that

$$
\mathrm{G}_{g} \vdash_{0} \operatorname{Nash}(a) \text { if and only if } a \in E_{g} .
$$

The right-hand side of (2.8) is a statement in the nonformalized language. Thus (2.8) relates the statement " $a$ is a Nash equilibrium" in the formalized language to the nonformalized extensional counterpart. This is translated into $\mathscr{P}^{\omega}$ as follows:

$$
\underset{y \in E_{g}}{\vee}\left(\bigwedge_{i=1}^{n} a_{i}=y_{i}\right)
$$

which is denoted by $\operatorname{Nash}^{E}(a)$. Of course, $\operatorname{Nash}(a)$ is the basic definition of Nash equilibrium, and $\operatorname{Nash}^{E}(a)$ is an extensional representation of it. Under Eq, this is also

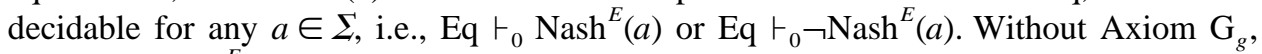
however, $\operatorname{Nash}^{E}(a)$ does not have the meaning "Nash equilibrium", i.e., neither Eq $\vdash_{0} \operatorname{Nash}^{E}(a) \supset \operatorname{Nash}(a)$ nor $\operatorname{Eq} \vdash_{0} \operatorname{Nash}(a) \supset \operatorname{Nash}^{E}(a)$. Under Eq and $G_{g}$, these two are equivalent, which is stated as Lemma 2.5.(1).

If we try to give an intensional definition of a subsolution, it would involve the second-order consideration, which is not allowed in our language. Hence we give the 
extensional definition of a subsolution in our formal language. We denote the following formula by $\operatorname{Sol}^{k}(a)$ :

$$
\underset{y \in E_{g}^{k}}{\vee}\left(\bigwedge_{i=1}^{n} a_{i}=y_{i}\right)
$$

for $k=1, \ldots, \sigma$. The formula $\operatorname{Sol}^{k}(a)$ describes " $a$ belongs to the subsolution $E_{g}^{k,}$.

\section{Lemma 2.5.}

1. $\mathrm{Eq}, \mathrm{G}_{g} \vdash_{0} \wedge_{x \in \Sigma}\left(\operatorname{Nash}(x) \equiv \operatorname{Nash}^{E}(x)\right)$;

2. Eq, $\mathrm{G}_{g} \vdash_{0} \wedge_{x}\left(\operatorname{Nash}(x) \equiv \vee_{k=1}^{\sigma} \operatorname{Sol}^{k}(x)\right)$.

We abbreviate $\wedge_{x \in \Sigma}$ as $\wedge_{x}$, etc. unless it is confusing.

Proof. We prove (1). Let $a$ be an arbitrary profile. Suppose $\mathrm{G}_{g} \vdash_{0} \operatorname{Nash}(a)$. Then $a \in E_{g}$ by (2.8). Thus Eq $\vdash_{0} \vee_{y \in E_{g}}\left(\wedge_{i} a_{i}=y_{i}\right)$, i.e., $\operatorname{Eq} \vdash_{0} \operatorname{Nash}^{E}(a)$. Since $\operatorname{Nash}(a)$ is decidable under $\mathrm{G}_{g}$ by (2.7), we have proved Eq, $\mathrm{G}_{g} \vdash_{0} \operatorname{Nash}(a) \supset \operatorname{Nash}^{E}(a)$. Noting that $\operatorname{Nash}^{E}(a)$ is decidable under Eq, we can repeat a similar argument to have Eq, $\mathrm{G}_{g} \vdash_{0} \operatorname{Nash}^{E}(a) \supset$ $\operatorname{Nash}(a)$. Thus Eq, $\mathrm{G}_{g} \vdash_{0} \operatorname{Nash}^{E}(a) \equiv \operatorname{Nash}(a)$. Since $a$ is an arbitrary profile, we have

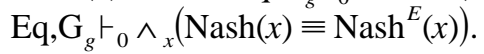

In Section 6, we need to assume that these equivalences are common knowledge among the players. If we assume that Axioms $\mathrm{Eq}$ and $\mathrm{G}_{g}$ are common knowledge, these equivalences are common knowledge, particularly, (2) becomes

$$
C(\mathrm{Eq}), C\left(\mathrm{G}_{g}\right) \vdash_{\omega}{ }_{x} C\left(\operatorname{Nash}(x) \equiv \underset{k=1}{\sigma} \operatorname{Sol}^{k}(x)\right) \text {. }
$$

This follows from Lemmas 2.5, 2.2.(2), 2.3.(1), and 2.4.(1). Note, however, that the common knowledge of the equivalence of $\operatorname{Nash}^{E}(a)$ and $\vee_{k=1}^{\sigma} \operatorname{Sol}^{k}(a)$ is provable without these axioms. Indeed, $\vdash_{0} \operatorname{Nash}^{E}(a) \equiv \vee_{k=1}^{\sigma} \operatorname{Sol}^{k}(a)$ for all $a \in \Sigma$, which this is simply extensional equivalence, and then $\vdash_{\omega} \wedge_{x} C\left(\operatorname{Nash}^{E}(x) \equiv \vee_{k=1}^{\sigma} \operatorname{Sol}^{k}(x)\right)$.

Note that Axioms Eq and $\mathrm{G}_{g}$ are consistent with respect to $\vdash_{0}$, i.e., there is no formula $A$ such that Eq, $\mathrm{G}_{g} \vdash_{0} \neg A \wedge A$. This can be proved by constructing a model of them by the Soundness Theorem for $\mathrm{GL}_{0}^{\mathrm{f}}$. It follow from this that $C(\mathrm{Eq})$ and $C\left(\mathrm{G}_{g}\right)$ are also consistent with respect to $\vdash_{\omega}$ by Lemma 2.2.(1), which we will use in Sections 5 and 6.

\section{Final decision axioms}

In a given game $g$, each player deliberates on his and the others' strategy choices and may reach some predictions on their final decisions. Now we describe these "predictions" by $n$-ary symbols $D_{1}, \ldots, D_{n}$, that is, each $D_{i}\left(a_{1}, \ldots, a_{n}\right)$ is intended to mean that player $i$ predicts that players $1, \ldots, n$ could choose $\left(a_{1}, \ldots, a_{n}\right)$ as their final decisions, where $a_{i}$ is his own decision. Note that each player's prediction may not be uniquely determined. 
The following are base axioms for $D_{1}(\cdot), \ldots, D_{n}(\cdot)$ : for each $i=1, \ldots, n$,

$\operatorname{Axiom~D1} 1_{i}^{0} \cdot \wedge_{x}\left(D_{i}(x) \supset \wedge_{y_{i}} R_{i}\left(x: y_{i} ; x_{-i}\right)\right)$;

$\operatorname{Axiom~} \mathbf{D 2}{ }_{i}^{0} \cdot \wedge_{x} \wedge_{j}\left(D_{i}(x) \supset D_{j}(x)\right)$

$\operatorname{Axiom~} \mathbf{D 3}_{i}^{0} \cdot \wedge_{x}\left(D_{i}(x) \supset K_{i}\left(D_{i}(x)\right)\right) ;$

Axiom $\mathbf{D 4}_{i}^{0} \cdot \wedge_{x} \wedge_{y} \wedge_{j}\left(D_{i}(x) \wedge D_{i}(y) \supset D_{i}\left(x_{j} ; y_{-j}\right)\right)$.

These are verbally as follows:

D $1_{i}^{0}$ : (Best Response to Predicted Decisions): If player $i$ predicts final decisions $x_{1}, \ldots, x_{n}$ for the players, then his own final decision $x_{i}$ maximizes his payoff against his prediction $x_{-i}$, that is, $x_{i}$ is a best response to $x_{-i}$.

D2 ${ }_{i}^{0}$ : (Identical Predictions): The other players reach the same predictions as player $i$ 's.

D3 ${ }_{i}^{0}$ : (Knowledge of Predictions): Player $i$ knows his own predictions.

D $4_{i}^{0}$ : (Interchangeability): In predicting the players' decisions, he assumes that the players are independent decision makers. This axiom states that when multiple predictions are possible, any combination of predictions on individual decisions is again a prediction profile. This may be better understood by looking at the equivalent formulation focussing on individual predictions (see Subsection 7.2).

These axioms are requirements for decision (prediction) making in the mind of each player $i=1, \ldots, n$. Hence we assume that these axioms are known to player $i$. These are described as $K_{i}\left(\mathrm{D} 1_{i}^{0}\right), K_{i}\left(\mathrm{D} 2_{i}^{0}\right), K_{i}\left(\mathrm{D} 3_{i}^{0}\right), K_{i}\left(\mathrm{D} 4_{i}^{0}\right)$. We denote these formulae by $\mathrm{D} 1_{i}$, $\mathrm{D} 2_{i}, \mathrm{D} 3_{i}, \mathrm{D} 4_{i}$, respectively. Now, the problem is whether these axioms determine "unknown" symbol $D_{i}(\cdot)$ in terms of the "primitives", $\mathbf{s}_{11}, \ldots, \mathbf{s}_{1 \ell_{1}} ; \ldots ; \mathbf{s}_{n 1}, \ldots, \mathbf{s}_{n \ell_{n}}$, $R_{1}, \ldots, R_{n}$ and $K_{1}, \ldots, K_{n}$. In the following, we denote $\mathrm{D} 1_{i}^{0} \wedge \ldots \wedge \mathrm{D} 4_{i}^{0}$ and $\mathrm{D} 1_{i} \wedge \ldots \wedge \mathrm{D} 4_{i}$ by $\mathrm{D}_{i}^{0}(1-4)$ and $\mathrm{D}_{i}(1-4)$.

We will take three steps to distil "solutions" from these axioms. Since the three steps are quite different, we give a brief explanation of these steps.

Step 0. The knowledge, $D_{i}(1-4)$, of the base axioms is incomplete as the requirement to determine $D_{i}(\cdot)$ 's, and the process of completing this knowledge forms an infinite regress of the knowledge of $\mathrm{D}_{1}(1-4), \ldots, \mathrm{D}_{n}(1-4)$. The resulting outcome of this infinite regress is described as the common knowledge of $\mathrm{D}_{1}(1-4), \ldots, \mathrm{D}_{n}(1-4)$, which we will denote by $C(\mathrm{D}(1-4))$.

In the other two steps, we "find solutions" of the common knowledge, $C(\mathrm{D}(1-4))$, of these axioms. It might be useful to remember a practice in middle-school mathematics that solving a simultaneous equation needs two steps: (1): assuming that a given equation has a solution, we calculate possible solutions from the equation; and (2): we 
verify that the solutions obtained satisfies the equation. In the same way, we have two steps to solve the common knowledge of the axioms as follows:

Step 1. We derive a necessary condition from the common knowledge $C(\mathrm{D}(1-4))$ of the above axioms. In Section 4, we will discuss Steps 0 and 1.

Step 2. We will verify that the necessary condition derived in Step 1 is a solution for $C(\mathrm{D}(1-4))$. In fact, this depends upon a game. In Section 5, we will treat games satisfying interchangeability (2.1). In Section 6, we consider games not satisfying (2.1), where some additional information is required to have a "solution".

In addition to these three steps, we will discuss, in Section 7, the status of each of the above base axioms in our axiomatization, their variants and the comparisons of Johansen (1982).

In the remaining of this section, we prepare some basic results on the axioms $\mathrm{D}_{1}(1-4), \ldots, \mathrm{D}_{n}(1-4)$.

Notice that $D_{j}(\cdot)$ 's occur in Axiom D2 for all $j$. These $D_{j}(\cdot)$ 's are determined by the other axioms $\mathrm{D}_{j}(1-4)$ 's. Then $D_{j}(\cdot)$ 's would be just symbols without meaning for player $i$ unless he knows the other axioms $\mathrm{D}_{j}(1-4)$ 's $(j \neq i)$. In other words, $\mathrm{D}_{j}(1-4)$ 's give operational meanings to the symbols $D_{j}(\cdot)$ 's. We need to give some operational knowledge of $D_{j}(\cdot)$ ', which is assumed here to be $\mathrm{D}_{j}(1-4)$ 's. In fact, this is the first step to the infinite regress to be discussed later.

The following proposition states that the addition of $\mathrm{D}_{j}(1-4)$ 's to $\mathrm{D}_{i}(1-4)$ is nontrivial.

Proposition 3.1. Let $i, j$ be distinct players. Then

1. neither $\mathrm{D}_{i}(1-4) \vdash_{\omega} \mathrm{D}_{j}^{0}(1-4)$ nor $\mathrm{D}_{i}(1-4) \vdash_{\omega} \neg \mathrm{D}_{j}^{0}(1-4)$;

2. neither $\mathrm{D}_{i}(1-4) \vdash_{\omega} \mathrm{D}_{j}(1-4)$ nor $\mathrm{D}_{i}(1-4) \vdash_{\omega} \neg \mathrm{D}_{j}(1-4)$;

3. neither $\mathrm{D}_{i}(1-4) \vdash_{\omega} K_{i}\left(\mathrm{D}_{j}(1-4)\right)$ nor $\mathrm{D}_{i}(1-4) \vdash_{\omega} \neg K_{i}\left(\mathrm{D}_{j}(1-4)\right)$.

Proof. We prove only the first assertion of (3). Suppose $\mathrm{D}_{i}(1-4) \vdash_{\omega} K_{i}\left(\mathrm{D}_{j}(1-4)\right)$, i.e., $\vdash_{\omega} \mathrm{D}_{i}(1-4) \supset K_{i}\left(\mathrm{D}_{j}(1-4)\right)$. By Lemma 2.2.(1), $\vdash_{0} \varepsilon \mathrm{D}_{i}(1-4) \supset \varepsilon K_{i}\left(\mathrm{D}_{j}(1-4)\right)$, which implies $\vdash_{0} \mathrm{D}_{i}^{0}(1,2,4) \supset \mathrm{D}_{j}^{0}(1,2,4)$, since $\varepsilon \mathrm{D} 3_{k}$ is equivalent to $\wedge_{x}\left(D_{k}(x) \supset D_{k}(x)\right)$. Hence $\vDash{ }_{\tau} \mathrm{D}_{i}^{0}(1,2,4) \supset \mathrm{D}_{j}^{0}(1,2,4)$ for any assignment $\tau$ by Soundness for $\mathrm{GL}_{0}^{\mathrm{f}}$. However, we can construct an assignment $\tau_{0}$ so that $\vDash{ }_{\tau_{0}} \mathrm{D}_{i}^{0}(1,2,4)$ but not $\vDash{ }_{\tau_{0}} \mathrm{D}_{j}^{0}(1,2,4)$, a contradiction. Therefore it is not the case that $\mathrm{D}_{i}(1-4) \vdash_{\omega} K_{i}\left(\mathrm{D}_{j}(1-\right.$ 4)).

We denote $\wedge_{j} \mathrm{D}_{j}(1-4)$ by $\mathrm{D}(1-4)$. Proposition 3.1 implies that for $D_{j}(\cdot)$ 's to be meaningful in Axiom $\mathrm{D} 2{ }_{i}$, we need to assume $K_{i}(\mathrm{D}(1-4))$. In fact, these are still insufficient to determine the meanings of all $\mathrm{D}_{j}(\cdot)$ 's in $K_{i}(\mathrm{D}(1-4))$, which will be discussed in Section 4. Here we state only the following undecidability:

$$
\text { neither } \mathrm{D}(1-4) \vdash_{\omega} K_{i}(\mathrm{D}(1-4)) \text { nor } \mathrm{D}(1-4) \vdash_{\omega} \neg K_{i}(\mathrm{D}(1-4)) \text {. }
$$


That is, the knowledge of $\mathrm{D}(1-4)$ is not derived from $\mathrm{D}(1-4)$ itself. In Section 4, we will give a general version of this claim, which leads to the infinite regress of the knowledge of $\mathrm{D}(1-4)$. The resulting outcome of this infinite regress would be expressed as the common knowledge of $\mathrm{D}(1-4)$.

In the following, we denote $\wedge_{j} \mathrm{D} t_{j}^{0}$ and $\wedge_{j} \mathrm{D} t_{j}$ by $\mathrm{D} t^{0}$ and $\mathrm{D} t$ for $t=1,2,3,4$, and $\mathrm{D} 1^{0} \wedge \mathrm{D} 2^{0}$ and $\mathrm{D} 1 \wedge \mathrm{D} 2 \wedge \mathrm{D} 4$ by $\mathrm{D}^{0}(1,2)$ and $\mathrm{D}(1,2,4)$, etc.

Lemma 3.2. $\mathrm{D}^{0}(1,2) \vdash_{0} \wedge_{x}\left(D_{i}(x) \supset \operatorname{Nash}(x)\right)$.

Proof. Let $a$ be an arbitrary profile. Since D $2^{0} \vdash_{0} D_{i}(a) \supset D_{j}(a)$ and $\mathrm{D} 1^{0} \vdash_{0} D_{j}(a) \supset$ $\wedge_{y_{j}} R_{j}\left(a_{j} ; a_{-j}: y_{j} ; a_{-j}\right)$ for all $i, j$, we have $\mathrm{D}^{0}(1,2) \vdash_{0} D_{i}(a) \supset \wedge_{y_{j}} R_{j}\left(a_{j} ; a_{-j}: y_{j} ; a_{-j}\right)$ for all $j$. Thus $\mathrm{D}^{0}(1,2) \vdash_{0} D_{i}(a) \supset \wedge_{j} \wedge_{y_{j}} R_{j}\left(a_{j} ; a_{-j}: y_{j} ; a_{-j}\right) \quad$ by $\wedge$-Rule, i.e., $\quad \mathrm{D}^{0}(1,2) \vdash_{0} D_{i}(a) \supset$ $\operatorname{Nash}(a)$. Hence we have the assertion.

Thus, Nash equilibrium is a necessary condition for Axioms $\mathrm{D}^{0}(1,2)$. In fact, we will show in our full axiomatization that $D_{i}(a)$ implies $C(\operatorname{Nash}(a))$, i.e., it is the common knowledge that $a$ is a Nash equilibrium. The following lemma is indicative of this common knowledge result.

Lemma 3.3. $\mathrm{D}(2,3) \vdash_{\omega} \wedge_{x}\left(D_{j}(x) \supset K_{i}\left(D_{k}(x)\right)\right)$ for any $i, j, k$ (i,j,k may be identical $)$.

Proof. Let $a$ be an arbitrary profile. Since D2 $\vdash_{\omega} K_{i}\left(D_{i}(a) \supset D_{k}(a)\right)$, we have D2 $\vdash_{\omega} K_{i}\left(D_{i}(a)\right) \supset K_{i}\left(D_{k}(a)\right)$ by $\mathrm{MP}_{i}$ and MP. Since D2 $\vdash_{\omega} D_{j}(a) \supset D_{i}(a)$ and D3 $\vdash_{\omega} D_{i}(a) \supset$ $K_{i}\left(D_{i}(a)\right)$, we have $\mathrm{D}(2,3) \vdash_{\omega} D_{j}(a) \supset K_{i}\left(D_{i}(a)\right)$. Hence we have $\mathrm{D}(2,3) \vdash_{\omega} D_{j}(a) \supset K_{i}\left(D_{k}(a)\right)$. This implies the assertion.

We prepare one more lemma, which will be used in the later sections.

\section{Lemma 3.4.}

1. $\varepsilon \mathrm{D}(1-4)$ is consistent with respect to $\vdash_{0}$, and $\varepsilon \mathrm{D}(1-4), D_{i}(a)$ is also consistent for any $a \in \Sigma$.

2. $\vdash_{\omega} \mathrm{D}(1-4)$ does not hold.

\section{Proof.}

1. $\varepsilon \mathrm{D}(1-4)$ is equivalent to $\mathrm{D}^{0}(1,2,4)$ with respect to $\vdash_{0}$. To prove the consistency of $\mathrm{D}^{0}(1,2,4)$, by Soundness for $\mathrm{GL}_{0}^{\mathrm{f}}$, it suffices to construct an assignment $\tau$ so that $\vDash{ }_{\tau} \mathrm{D}^{0}(1,2,4)$. Define $\tau$ so that $\tau\left(D_{i}(a)\right)=$ false for all profiles $a$ and $i=1, \ldots, n$. Then $\vDash{ }_{\tau} \mathrm{D}^{0}(1,2,4)$ in the trivial sense. The latter is proved by modifying $\tau$.

2. Suppose $\vdash_{\omega} \mathrm{D}(1-4)$. Then $\vdash_{0} \varepsilon \mathrm{D}(1-4)$, i.e., $\vdash_{0} \mathrm{D}^{0}(1,2,4)$, by Lemma 2.2.(1). However, we can construct $\tau$ in a similar manner as in (1) so that $\mathrm{D}^{0}(1,2,4)$ is false, which together with (2.4) implies not $\vdash_{0} \mathrm{D}^{0}(1,2,4)$. Thus $\vdash_{\omega} \mathrm{D}(1-4)$ is not the case. 


\section{Infinite regress of the knowledge of the axioms and its evaluations}

In this section, we show that the process of making Axioms D1 to D4 meaningful forms an infinite regress of the knowledge of $\mathrm{D}(1-4)$. The resulting outcome of this infinite regress is expressed as the common knowledge of $\mathrm{D}(1-4)$. We make prooftheoretical evaluations of this infinite regress.

\subsection{Infinite regress of the knowledge of the final decision axioms}

It could be found by looking at Lemma 3.3 carefully that axioms D1-D4 require each player to know them. Lemma 3.3 states that each player knows any other players' predictions on final decisions, but this would not make sense unless the meaning of "predictions on final decisions" is given to the players. In fact, the meaning is determined by the above four axioms themselves. Therefore each player needs to know them, i.e., $K_{i}(\mathrm{D}(1-4))$ for $i=1, \ldots, n$.

Once the players know these axioms, each knows the consequences from these axioms such as the assertion of Lemma 3.2, i.e., $\left.\wedge_{j} K_{j}(\mathrm{D}(1-4)) \vdash_{\omega} \wedge_{x} K_{i}\left(D_{i}(x) \supset \mathrm{Nash}(x)\right)\right)$. It is, however, more to the point that the following is provable: for any $l, k=1, \ldots, n$,

$$
\bigwedge_{j} K_{j}(\mathrm{D}(1-4)) \vdash_{\omega} \bigwedge_{x}\left(D_{i}(x) \supset K_{l} K_{k}\left(D_{i}(x)\right)\right)
$$

In (4.1), the "imaginary" player $k$ in the mind of player $l$ knows that $x$ is a profile of predicted decisions. This imaginary player $k$ is not given the operational meaning, $\mathrm{D}(1-4)$, of "final decisions", though "real" $k$ is assumed to know $\mathrm{D}(1-4)$. This means that (4.1) does not make sense for the imaginary $k$. Thus we need to assume $K_{l} K_{k}(\mathrm{D}(1-4))$ : we reach the assumption set $\left\{L(\mathrm{D}(1-4)): L \in \bigcup_{t \leq 2} K(t)\right\}^{5}$. Once we assume this set of axioms, we would again meet the problem parallel to that arose in (4.1), that is, it holds that for any $K \in K(3)$,

$$
\left\{L(\mathrm{D}(1-4)): L \in \bigcup_{t \leq 2} K(t)\right\} \vdash_{\omega}{ }_{x}\left(D_{i}(x) \supset K\left(D_{i}(x)\right)\right)
$$

If we assume the knowledge of $\mathrm{D}(1-4)$ up depth 2, the knowledge of depth 3 is necessarily involved. The imaginary players in the epistemic world of depth 3 should know $\mathrm{D}(1-4)$.

In general, we have the following proposition.

Proposition 4.1. For any players $i, j$, finite $m \geq 0$ and $K \in K(m+1)$,

$$
\left\{L(\mathrm{D}(2,3)): L \in \bigcup_{t \leq m} K(t)\right\} \vdash_{\omega} \bigwedge_{x}\left(D_{i}(x) \supset K\left(D_{j}(x)\right)\right)
$$

\footnotetext{
${ }^{5}$ By Axiom $\mathrm{T}_{i}$, it suffices to assume $\{L(\mathrm{D}(1-4)): L \in K(2)\}$. However, we keep some redundancies for presentational consistency.
} 
Proof. Let $a$ be an arbitrary profile. We prove that for any $K \in K(m+1),\{L(\mathrm{D}(2,3)): L \in$ $\left.\bigcup_{t \leq m} K(t)\right\} \vdash{ }_{\omega} D_{i}(a) \supset K\left(D_{j}(a)\right)$. For $m=0$, this is Lemma 3.3. Now we assume the induction hypothesis that the assertion holds for $m$. Let $K=K^{\prime} K_{k} \in K(m+2)$. Then $K^{\prime} \in K(m+1)$. By the induction basis and some applications of Nec, MP and $\mathrm{MP}_{i}$, $K^{\prime}(\mathrm{D}(2,3)) \vdash_{\omega} K^{\prime}\left(D_{i}(a) \supset K_{k}\left(D_{j}(a)\right)\right)$, and then $K^{\prime}(\mathrm{D}(2,3)) \vdash_{\omega} K^{\prime}\left(D_{i}(a)\right) \supset K^{\prime} K_{k}\left(D_{j}(a)\right)$. This together with the induction hypothesis implies $\left\{L(\mathrm{D}(2,3)): L \in \bigcup_{t \leq m+1} K(t)\right\} \vdash_{\omega} D_{i}(a) \supset$ $K^{\prime} K_{k}\left(D_{j}(a)\right)$.

Thus, if we assume the knowledge of Axiom $\mathrm{D}(1-4)$ up to depth $m$, the knowledge of depth $m+1$ is necessarily involved. Hence the knowledge of $\mathrm{D}(1-4)$ up to depth $m+1$ should be added. The following theorem states that this addition is inevitable, which is a general version of (3.1). This will be proved in Subsection 4.2.

Theorem 4.A. For any finite $m \geq 0$ and for any $K \in \bigcup_{t>m} K(t)$,

$$
\begin{aligned}
& \text { neither }\left\{L(\mathrm{D}(1-4)): L \in \bigcup_{t \leq m} K(t)\right\} \vdash_{\omega} K(\mathrm{D}(1-4)) \\
& \operatorname{nor}\left\{L(\mathrm{D}(1-4)): L \in \bigcup_{t \leq m} K(t)\right\} \vdash_{\omega} \neg K(\mathrm{D}(1-4)) .
\end{aligned}
$$

This theorem states that without assuming $K(\mathrm{D}(1-4))$ for all $K$ of all depths, some imaginary players living in the mind of the players in some depth could not know the definition of $D_{i}(\cdot)$ 's. To avoid this problem, we should assume

$$
\left\{K(\mathrm{D}(1-4)): K \in \bigcup_{t<\omega} K(t)\right\}
$$

Thus we meet an infinite regress of the knowledge of axioms $\mathrm{D}(1-4)$. The infinite regress leads to the set of (4.4), and its conjunction is the common knowledge of $\mathrm{D}(1-4)$. We will adopt this common knowledge formula as an axiom and consider its implications. The following result holds, which was given in Kaneko-Nagashima (1991) and (1996). For completeness, we will give a brief proof. In the following, when we write $D_{i}(\cdot), D_{j}(\cdot)$ without quantification of $i, j$, they are arbitrary players.

\section{Proposition 4.2.}

1. $C(\mathrm{D}(2,3)) \vdash_{\omega} \wedge_{x}\left(D_{i}(x) \supset C\left(D_{j}(x)\right)\right)$.

2. $C(\mathrm{D}(1-3)) \vdash_{\omega} \wedge_{x}\left(D_{i}(x) \supset C(\operatorname{Nash}(x))\right)$.

Proof. (1) follows Proposition 4.1. Consider (2). Let $a$ be an arbitrary profile. First, $\mathrm{D}(1,2) \vdash_{0} D_{i}(a) \supset \operatorname{Nash}(a)$ by Lemma 3.2. Hence, by Lemma 2.2.(1), we have $C(\mathrm{D}(1,2)) \vdash_{\omega} C\left(D_{i}(a) \supset \mathrm{Nash}(a)\right), \quad$ and $C(\mathrm{D}(1,2)) \vdash_{\omega} C\left(D_{i}(a)\right) \supset C(\mathrm{Nash}(a))$ by Lemma 2.3.(1). Since $C(\mathrm{D}(2,3)) \vdash_{\omega} D_{i}(a) \supset C\left(D_{i}(a)\right)$ by (1), we have $C(\mathrm{D}(1-3)) \vdash_{\omega} D_{i}(a) \supset$ $C(\mathrm{Nash}(a))$. 
In fact, $C(\operatorname{Nash}(x))$ will be shown to be the solution of $C(\mathrm{D}(1-4))$ for a game satisfying interchangeability (2.1), which will be the subject of Section 5. Here, we can ask whether the conclusion of Proposition 4.2.(2) is provable from the knowledge of $D(1-4)$ up to some finite depth. The following theorem states the negative answer. Since this can be proved in the same manner as in the proof of Theorem 4.A, we omit the proof.

Theorem 4.B. For any finite $m$,

$$
\begin{aligned}
& \text { neither }\left\{L(D(1-4)): L \in \bigcup_{t \leq m} K(t)\right\} \vdash_{\omega}{\underset{x}{x}}\left(D_{i}(x) \supset C(\operatorname{Nash}(x))\right) \\
& \text { nor }\left\{L(D(1-4)): L \in \bigcup_{t \leq m} K(t)\right\} \vdash_{\omega} \neg \wedge_{x}\left(D_{i}(x) \supset C(\operatorname{Nash}(x))\right) .
\end{aligned}
$$

The above derivation of the infinite regress, a fortiori, $C(\mathrm{D}(1-4))$, is still heuristic. However, it can be formulated in the following manner, whose proof is also omitted.

Theorem 4.C. Let $\Gamma$ be a set of formulae. Suppose that $\Gamma \vdash_{\omega} \vee_{x} K\left(D_{i}(x)\right) \supset K(\mathrm{D}(1-4))$ for all $K \in \bigcup_{m<\omega} K(m)$. Then $\Gamma \vdash_{\omega} \vee_{x} D_{i}(x) \supset C(\mathrm{D}(1-4))$.

In Subsection 4, we show that this set $\Gamma$ needs to contain some common knowledge.

The results given in this section are still purely solution-theoretic: they do not require the players to know the game, i.e., neither Axioms Eq nor $\mathrm{G}_{g}$. The knowledge of the structure of the game will be needed in Sections 5 and 6 .

\subsection{Evaluations of the infinite regress}

To prove the undecidability theorems given in the above subsection, we need the depth $\delta(A)$ of a formula $A$. Using this concept, we will evaluate the provability of an epistemic statement.

We define the depth $\delta(A)$ by induction on the structure of a formula from the inside:

$(0): \delta(A)=\emptyset$ for any atomic $A$;

(1): $\delta(\neg A)=\delta(A)$;

(2): $\delta(A \supset B)=\delta(A) \cup \delta(B)$;

(3): $\delta(\wedge \Phi)=\delta(\vee \Phi)=\bigcup_{A \in \Phi} \delta(A)$;

(4): $\delta\left(K_{j}(A)\right)= \begin{cases}\{(j)\} & \text { if } \delta(A)=\emptyset \\ \left\{\left(j, i_{1}, \ldots, i_{m}\right):\left(i_{1}, \ldots, i_{m}\right) \in \delta(A) \text { and } j \neq i_{1}\right\} \cup & \\ \left\{\left(i_{1}, \ldots, i_{m}\right):\left(i_{1}, \ldots, i_{m}\right) \in \delta(A) \text { and } j=i_{1}\right\} & \text { otherwise. }\end{cases}$

For any set $\Gamma$ of formulae, let $\delta(\Gamma)$ be $\bigcup_{A \in \Gamma} \delta(A)$. Define $\sup \delta(\Gamma)=$ $\sup \left\{m:\left(i_{1}, \ldots, i_{m}\right) \in \delta(\Gamma)\right\}$. For example, $\delta\left(\mathrm{D} 3_{i}^{0}\right)=\delta\left(\mathrm{D} 1_{i}\right)=\{(i)\}, \delta(\mathrm{D} 3)=\{(i): i=1, \ldots, n\}$ and $\delta\left(\wedge_{i} K_{i}(\mathrm{D}(1-4))\right)=\{(i, j): i \neq j\}$.

The following lemma is the key to prove the undecidability results presented, and is 
proved in the Gentzen-style sequent calculus formulation of $\mathrm{GL}_{\omega}$ in Kaneko (1998) using the cut-elimination theorem for $\mathrm{GL}_{\omega}{ }^{6}$

Lemma 4.3* (Depth Lemma). Let $K=K_{i_{1}} \ldots K_{i_{m}} \in K(m), \Gamma$ a set of formulae and $A$ a formula. Assume $\Gamma \vdash_{\omega} K(A)$. Assume $\left(i_{1}, \ldots, i_{m}\right) \notin \delta(\Gamma)$.

1. Let $\sup \delta(\Gamma \cup\{A\})<\omega$. Then (a) $\Gamma$ is inconsistent with respect to $\vdash_{\omega}$; or (b) $\vdash_{\omega} A$.

2. (a) $\varepsilon \Gamma$ is inconsistent with respect to $\vdash_{0}$; or (b) $\vdash_{\omega} \varepsilon A$.

The first states that if $K_{i_{1}} \ldots K_{i_{m}}(A)$ is derived from the set of premises $\Gamma$, then $\Gamma$ is inconsistent or $A$ is a trivial formula. The second is essentially the same, but needs some modification for some technical difficulty if $\sup \delta(\Gamma \cup\{A\})$ is infinite.

We use this lemma to prove Theorems 4.A.

Proof of Theorem 4.A. Let us start with the proof of (4.3). Suppose, on the contrary, that $\left\{L(\mathrm{D}(1-4)): L \in \bigcup_{t \leq m} K(t)\right\} \vdash_{\omega} \neg K(\mathrm{D}(1-4))$. Then $\quad \varepsilon \mathrm{D}(1-4) \vdash_{0} \neg \varepsilon \mathrm{D}(1-4)$ by Lemma 2.2.(1), which contradicts Lemma 3.4.(1).

Now consider (4.2). Suppose $\left\{L(\mathrm{D}(1-4)): L \in \bigcup_{t \leq m} K(t)\right\} \vdash_{\omega} K(\mathrm{D}(1-4))$ for some $K=K_{i_{1}} \ldots K_{i_{\ell}}$ and $\ell>m$. Then since $K(\mathrm{D}(2,3)) \vdash_{\omega} K\left(D_{i}(a)\right) \supset K K_{j}\left(D_{i}(a)\right)$ for any $j$ and profile $a$, we have $\left\{L(\mathrm{D}(1-4)): L \in \bigcup_{t \leq m} K(t)\right\} \vdash_{\omega} D_{i}(a) \supset K K_{j}\left(D_{i}(a)\right)$. Let $j$ be different from the index of the innermost symbol of $K$. This is written as

$$
\left\{L(D(1-4)): L \in \bigcup_{t \leq m} K(t)\right\}, D_{i}(a) \vdash_{\omega} K K_{j}\left(D_{i}(a)\right) .
$$

Since $\sup \delta\left(\left\{L(\mathrm{D}(1-4)): L \in \bigcup_{t \leq m} K(t)\right\} \cup\left\{D_{i}(a)\right\}\right)=m+1$, it follows from Lemma 4.3. (1) that either $\left\{L(\mathrm{D}(1-4)): L \in \bigcup_{t \leq m} K(t)\right\} \cup\left\{D_{i}(a)\right\}$ is inconsistent or $\vdash_{\omega} D_{i}(a)$. In the former case, $\varepsilon \mathrm{D}(1-4), D_{i}(a)$ is inconsistent with respect to $\vdash_{0}$, which is impossible by Lemma 3.4.(1). In the second case, we have $\vdash_{0} D_{i}(a)$, which is also impossible.

The following is the result corresponding to Theorem 4.C.

Theorem 4.D. Let $\Gamma$ be a set of formulae with $\sup \delta(\Gamma)<\omega$, and assume that $\Gamma \vee\left\{\vee_{x} D_{i}(x)\right\}$ is consistent with respect to $\vdash_{\omega}$. Then $\Gamma \vdash_{\omega} \vee_{x} D_{i}(x) \supset C(\mathrm{D}(1-4))$ does not hold.

Proof. On the contrary, suppose $\Gamma \vdash_{\omega} \vee_{x} D_{i}(x) \supset C(\mathrm{D}(1-4))$. This is equivalent to that $\vdash_{\omega}(\wedge \Phi) \wedge\left(\vee_{x} D_{i}(x)\right) \supset C(\mathrm{D}(1-4))$ for some finite subset $\Phi$ of $\Gamma$. Let $K \in K(m)$ for $m>\sup \delta(\Gamma)$. Then $\vdash_{\omega}(\wedge \Phi) \wedge\left(\vee_{x} D_{i}(x)\right) \supset K(\mathrm{D}(1-4))$. The application of Lemma

\footnotetext{
${ }^{6}$ This lemma is an extension of the depth lemma proved in Kaneko-Nagashima (1997b) for the propositional epistemic logic S4 based the cut-elimination theorem obtained by Ohnishi-Matsumoto (1957).
} 
4.3.(1) to this statement implies that either $(\wedge \Phi) \wedge\left(\vee_{x} D_{i}(x)\right)$ is inconsistent with respect to $\vdash_{\omega}$ or $\vdash_{\omega} \mathrm{D}(1-4)$. The former is impossible by the assumption of the theorem. The latter is also impossible by Lemma 3.4.(2).

This theorem implies that infinite depth is necessarily involved for $\Gamma$ in Theorem 4.C. In fact, we can prove the knowledge structure of common knowledge is exactly involved in $\Gamma$. However, since it needs more detailed argument, we omit it.

\section{Solvable games}

In Section 4, we derived the common knowledge, $C(\mathrm{D}(1-4))$, of Axioms $\mathrm{D}(1-4)$. We adopt this $C(\mathrm{D}(1-4))$ as an axiom and consider its implications. It was shown in Kaneko-Nagashima (1991) and (1996) that this determines the final decision predicate $D_{i}(a)$ to be $C(\operatorname{Nash}(a))$ under the common knowledge of interchangeability (2.1). Here we will evaluate epistemic aspects of this result, and consider also the playability of a game.

\subsection{Determination of the final decision prediction and its evaluations}

Proposition 4.2.(2) states that under $C(\mathrm{D}(1-4)), D_{i}(a)$ implies $C(\operatorname{Nash}(a))$. Axiom D4 requires $D_{i}(\cdot)$ to be interchangeable, while $C(\mathrm{Nash}(\cdot))$ is not necessarily interchangeable. Hence $C(\operatorname{Nash}(a))$ may not capture all the properties of $C(\mathrm{D}(1-4))$. However, if we assume that interchangeability,

$$
\wedge_{x} \bigwedge_{y} \bigwedge_{j}\left(\operatorname{Nash}(x) \wedge \operatorname{Nash}(y) \supset \operatorname{Nash}\left(y_{j} ; x_{-j}\right)\right)
$$

is common knowledge, then $C(\operatorname{Nash}(\cdot))$ can be regarded as capturing all the properties of $C(\mathrm{D}(1-4))$. This corresponds to the verification step of the obtained solutions for a simultaneous equation, discussed in Section 3.

We denote the formula of (5.1) by Int, which is the formalized statement of (2.1). When a game $g$ satisfies (2.1), it holds that $\mathrm{G}_{g} \vdash_{0}$ Int, and by Proposition 2.2.(2), we have $C\left(\mathrm{G}_{g}\right) \vdash_{\omega} C(\mathrm{Int})$.

The following proposition states that $C(\operatorname{Nash}(\cdot))$ as $D_{i}(\cdot)$ 's satisfies our axioms.

\section{Proposition 5.1.}

1. $\vdash_{\omega} C(\mathrm{D}(1-3)[C(\mathrm{Nash})])$;

2. $C($ Int $) \vdash_{\omega} C(\mathrm{D} 4[C(\mathrm{Nash})])$,

where $\mathrm{D}(1-3)[C(\mathrm{Nash})]$ and $\mathrm{D} 4[C(\mathrm{Nash})]$ are the formulae obtained from $\mathrm{D}(1-3)$ and D4 by substituting $C(\operatorname{Nash}(a))$ for every occurrence of $D_{i}(a)(a \in \Sigma$ and $i=1, \ldots, n)$ in $\mathrm{D}(1-3)$ and $\mathrm{D} 4$. 


\section{Proof.}

1. We prove only $\vdash_{\omega} C(\mathrm{D} 3[C(\mathrm{Nash})])$. Since $\vdash_{\omega} C(\operatorname{Nash}(a)) \supset K_{i}(C(\operatorname{Nash}(a)))$ for all $i=1, \ldots, n$ by $(2.3)$, we have $\vdash_{\omega} \wedge_{i} \wedge_{x}\left[C(\operatorname{Nash}(x)) \supset K_{i}(C(\operatorname{Nash}(x)))\right]$, i.e., $\vdash_{\omega} \mathrm{D} 3[C(\mathrm{Nash})]$. By Lemma 2.2.(2), $\vdash_{\omega} C(\mathrm{D} 3[C(\mathrm{Nash})])$.

2. Since Int $\vdash_{0} \operatorname{Nash}(a) \wedge \operatorname{Nash}(b) \supset \operatorname{Nash}\left(b_{j} ; a_{-j}\right)$, we have $C(\operatorname{Int}) \vdash_{\omega} C[\operatorname{Nash}(a)$ $\left.\wedge \operatorname{Nash}(b) \supset \operatorname{Nash}\left(b_{j} ; a_{-j}\right)\right]$ by Lemma 2.2.(2). By Lemmas 2.3.(1) and 2.4.(1), $C(\mathrm{Int}) \vdash_{\omega} C(\operatorname{Nash}(a)) \wedge C(\operatorname{Nash}(b)) \supset C\left(\operatorname{Nash}\left(b_{j} ; a_{-j}\right)\right)$. Since $a, b, j$ are arbitrary,

$$
C(\mathrm{Int}) \vdash_{\omega} \wedge_{x} \hat{y}_{y} \bigwedge_{j}\left[C(\operatorname{Nash}(x)) \wedge C(\operatorname{Nash}(y)) \supset C\left(\operatorname{Nash}\left(y_{j} ; x_{-j}\right)\right)\right] .
$$

This is $C($ Int $) \vdash_{\omega} \quad \mathrm{D} 4[C(\mathrm{Nash})]$. By Lemma 2.2.(2), we have $C($ Int $) \vdash{ }_{\omega} C(\mathrm{D} 4[C(\mathrm{Nash})])$.

Thus, $C(\mathrm{Nash}(\cdot))$ is a solution of $C(\mathrm{D}(1-4))$ under $C(\mathrm{Int})$. Conversely, it can be proved in the same way as Proposition 4.2.(2) that

$$
C(\mathrm{D}(1,2,3)[\mathscr{A}]) \vdash_{\omega}{ }_{x} \wedge\left(A_{i}(x) \supset C(\operatorname{Nash}(x))\right) \text { for } i=1, \ldots, n,
$$

where $\mathscr{A}=\left\{\left(A_{1}(a), \ldots, A_{n}(a)\right): a \in \Sigma\right\}$ is any set of profiles of formulae indexed by $a \in \Sigma$. Thus $C(\operatorname{Nash}(\cdot))$ is weaker than any formulae satisfying $C(\mathrm{D}(1,2,3))$. Additionally, Proposition 5.1 states that $C(\operatorname{Nash}(\cdot))$ satisfies $C(\mathrm{D}(1-4))$ under $C($ Int $)$. Hence $C(\mathrm{Nash}(\cdot))$ is the deductively weakest formula among those satisfying $C(\mathrm{D}(1-4))$. We are looking for the deductively weakest formula for $D_{i}(\cdot)$, since it contains no additional information other than what we intend to describe by $C(\mathrm{D}(1-4))$.

The explicit formulation of the choice of the deductively weakest formula is given as the following axiom schemata:

$$
\text { (WD): } C(\mathrm{D}(1-4)[\mathscr{A}]) \wedge C\left(\wedge_{i} \wedge_{x}\left[D_{i}(x) \supset A_{i}(x)\right]\right) \supset \wedge_{i} \wedge_{x}\left[D_{i}(x) \equiv A_{i}(x)\right]
$$

where $\mathscr{A}=\left\{\left(A_{1}(a), \ldots, A_{n}(a)\right): a \in \Sigma\right\}$ is any set of vectors of formulae indexed by $a \in \Sigma$. Although, in fact, we should, probably, regard $C$ (WD) as our axiom, WD suffices for all the results in the subsequent analysis. Therefore we use simply WD instead of $C(\mathrm{WD})$.

Since Proposition 4.2.(2) and Proposition 5.1 imply $C(D(1-4)), C($ Int $) \vdash_{\omega} C(\mathrm{D}(1-$ $4)[C(\mathrm{Nash})]) \wedge C\left(\wedge_{x}\left(D_{i}(x) \supset C(\operatorname{Nash}(x))\right)\right)$. Since this is the premise of an instance of $\mathrm{WD}$, we obtain the following theorem.

Theorem 5.A. $C(\mathrm{D}(1-4)), C(\mathrm{Int}), \mathrm{WD} \vdash_{\omega} \wedge_{x}\left(D_{i}(x) \equiv C(\mathrm{Nash}(x))\right)$.

Recall that $C\left(\mathrm{G}_{g}\right) \vdash_{\omega} C$ (Int) for any game $g$ satisfying interchangeability (2.1). Hence it follows from Theorem 5.A that for a game $g$ satisfying (2.1),

$$
C(\mathrm{D}(1-4)), C\left(G_{g}\right), W D \vdash_{\omega} \hat{x}_{x}\left(D_{i}(x) \equiv C(\mathrm{Nash}(x))\right) .
$$

Thus, the above solution-theoretical result does not differentiate the abstract knowledge, $C$ (Int), on a game from the specific knowledge, $C\left(\mathrm{G}_{g}\right)$, of the game. However, these are 
differentiated by the existence-playability consideration, which is the subject of Subsection 5.2.

Now we evaluate the above procedure of unique determination. First, we ask whether any formula weaker than $C(\operatorname{Nash}(a))$ satisfies our axioms. In fact, for $\mathrm{D}(1,2)$ or $\mathrm{D}(1,2,4)$, we can replace $C(\operatorname{Nash}(\cdot))$ by $\operatorname{Nash}(\cdot)$, i.e., the following hold:

$$
\vdash_{\omega} C(\mathrm{D}(1,2)[\mathrm{Nash}]) \text {; and } C(\mathrm{Int}) \vdash_{\omega} C(\mathrm{D} 4[\mathrm{Nash}]) \text {. }
$$

Thus $C(\mathrm{D}(1,2,4))$ does not require the common knowledge operator $C(\cdot)$ for $\mathrm{Nash}(\cdot)$. The common knowledge is required by D3 together with D2, which is stated in the following theorem.

Theorem 5.B. Let $\mathscr{A}=\left\{\left(A_{1}(a), \ldots, A_{n}(a)\right): a \in \Sigma\right\}$ be a set of profiles of formulae.

1. If $\vdash_{\omega} \mathrm{D}(2,3)[\mathscr{A}]$, then $\vdash_{\omega} A_{i}(a) \supset C\left(A_{i}(a)\right)$ for any $i$ and $a$.

2. Suppose $\max _{i, a} \sup \delta\left(A_{i}(a)\right)<\omega$. Then $\vdash_{\omega} \mathrm{D}(2,3)[\mathscr{A}]$ if and only if $\vdash_{\omega} \neg A_{i}(a)$ or $\vdash_{\omega} A_{i}(a)$ for any $i$ and $a$.

\section{Proof.}

1. Suppose $\vdash_{\omega} \mathrm{D}(2,3)[\mathscr{A}]$. It can be proved in the same way as the proof of Lemma 3.3 that $\vdash_{\omega} \mathrm{D}(2,3)[\mathscr{A}] \supset\left(A_{i}(a) \supset K_{j}\left(A_{i}(a)\right)\right)$ for all $i, j$ and $a$. Hence $\vdash_{\omega} A_{i}(a) \supset K_{j}\left(A_{i}(a)\right)$ for all $i, j$ and $a$. From this together with Nec, MP and $\mathrm{MP}_{i}$, we have $\vdash_{\omega} A_{i}(a) \supset$ $K\left(A_{i}(a)\right)$ for all $K \in \bigcup_{t<\omega} K(t)$. Hence $\vdash_{\omega} A_{i}(a) \supset C\left(A_{i}(a)\right)$ by $\wedge$-Rule.

2. The if part is straightforward. The only-if part is proved as follows: Consider an arbitrary $A_{i}(a)$. Since $\sup \delta\left(A_{i}(a)\right)<\omega$, we can choose $K \in K(m)$ so that $m>$ $\sup \delta\left(A_{i}(a)\right)$. Since $\vdash_{\omega} A_{i}(a) \supset K\left(A_{i}(a)\right)$ by (1), Lemma 4.3.(1) states that $\vdash_{\omega} \neg A(a)$ or $\vdash_{\omega} A(a)$.

Theorem 5.B.(1) states that if some formulae satisfy D2 and D3, then they include common knowledge. Then (2) implies that if nontrivial formulae satisfy D2 and D3, they have infinite depths.

\subsection{Playability of and the knowledge of a game}

The introduction of an epistemic structure enables us to consider the problem of playability. In our context, the playability of a game is formulated as $\vee_{x} D_{i}(x)-$ the existence of predicted final decisions. According to Theorem 5.A, the question is equivalent to whether or not $\vee_{x} C(\operatorname{Nash}(x))$ is obtained from some axioms. This is different from $C\left(\vee_{x} \operatorname{Nash}(x)\right)$. The former states that there is some strategy profile $x$ such that it is common knowledge that $x$ is a Nash equilibrium, but the second states that the existence of a Nash equilibrium is common knowledge.

The solvability of a game is formulated as

$$
\operatorname{Int} \wedge\left({ }_{x}^{\vee} \operatorname{Nash}(x)\right)
$$


which we denote by Solv. Since $C$ (Solv) contains the existence of a Nash equilibrium, we have $C($ Solv $) \vdash_{\omega} C\left(\vee_{x} \mathrm{Nash}(x)\right)$. Nevertheless, this together with $C(\mathrm{D}(1-4))$,WD does not imply $\vee_{x} D_{i}(x)$ : Instead, we need to have some $\Gamma$ so that

$$
C(\mathrm{D}(1-4)), \mathrm{WD}, \Gamma \vdash_{\omega}{ }_{x}^{\vee} C(\mathrm{Nash}(x)) .
$$

We prove that $C\left(\right.$ Solv) is not sufficient as $\Gamma$ to have $\vee_{x} D_{i}(x)$, but $C\left(\mathrm{G}_{g}\right)$ guarantees playability when $g$ is solvable. Note that if $g$ is solvable, then $C\left(\mathrm{G}_{g}\right) \vdash_{\omega} C($ Int $)$.

\section{Theorem 5.C.}

1. Neither $C(\mathrm{D}(1-4)), \mathrm{WD}, C($ Solv $) \vdash_{\omega} \vee_{x} D_{i}(x)$ nor $C(\mathrm{D}(1-4)), \mathrm{WD}, C(\mathrm{Solv}) \vdash_{\omega} \neg \vee_{x} D(x)$.

2. Let $g$ be a solvable game. Then $C(\mathrm{D}(1-4)), \mathrm{WD}, C\left(\mathrm{G}_{g}\right) \vdash_{\omega} \vee_{x} D(x)$.

Thus $C\left(\mathrm{G}_{g}\right)$ suffices for $\Gamma$ in (5.4) with the condition that $g$ is a solvable game, but $C$ (Solv) is not sufficient. The significance of this theorem is to demarcate the knowledge of abstract conditions on the game from the knowledge of the specific structure of a game. Abstract treatments are convenient for our (investigators') considerations, but the specific knowledge of the game is needed for the players to play the game.

The first assertion of Theorem 5.C corresponds to the undecidability result presented in Kaneko-Nagashima (1996) that there is a specific three-person game with a unique Nash equilibrium in mixed strategies such that the playability statement is undecidable, while the common knowledge of the existence of a Nash equilibrium is provable under the common knowledge of real closed field axioms. Their undecidability is caused by the choice of a language the players use. Contrary to theirs, our unplayability is caused by the fact that a game is given abstractly and is not specified enough for the players. The second assertion states that once a game is fully specified for them, our undecidability is removed when it is solvable.

In fact, the playability and existence of a Nash equilibrium could not be distinguished without epistemic structures. Ignoring Axiom D3, Theorem 5.A is stated as follows:

$$
C(\mathrm{D}(1,2,4)), C(\mathrm{Int}), \mathrm{WD}(1,2,4) \vdash_{\omega}{ }_{x}^{\wedge}\left(D_{i}(x) \equiv \mathrm{Nash}(x)\right),
$$

where $\mathrm{WD}(1,2,4)$ is the corresponding modification of WD. In this case, $\vee_{x} D_{i}(x)$ is equivalent to $v_{x} \operatorname{Nash}(x)$. Hence

$$
C(\mathrm{D}(1,2,4)), C(\text { Solv }), \mathrm{WD}(1,2,4) \vdash_{\omega} \bigvee_{x} D_{i}(x)
$$

Thus the abstract existence knowledge leads to $\vee_{x} D_{i}(x)$, contrary to Theorem 5.C.(1).

The first assertion of (1) needs a long proof, but (2) can be proved with what we have already prepared. Therefore we give the proofs of those assertions in the reverse order.

Proof of (2). Since $g$ is a solvable game, it has a particular Nash equilibrium $a$. Then $\mathrm{G}_{g}$ $\vdash_{0} \operatorname{Nash}(a)$ by (2.7), which implies $C\left(\mathrm{G}_{g}\right) \vdash_{\omega} C(\mathrm{Nash}(a))$ by Lemma 2.2.(2). By Theorem 5.A, we have $C(\mathrm{D}(1-4)), \mathrm{WD}, C\left(\mathrm{G}_{g}\right) \vdash_{\omega} D_{i}(a)$. Hence $C(\mathrm{D}(1-4)), \mathrm{WD}, C\left(\mathrm{G}_{g}\right) \vdash_{\omega} \vee$ ${ }_{x} D_{i}(x)$. 
Lemma 5.2. Let $A$ be a formula including no $D_{1}, \ldots, D_{n}$. If $C(\mathrm{D}(1-4)), \mathrm{WD}, C(\mathrm{Solv}) \vdash_{\omega} A$, then $C(\mathrm{Solv}) \vdash_{\omega} A$.

Proof. Suppose $C(\mathrm{D}(1-4)), \mathrm{WD}, C($ Solv $) \vdash_{\omega} A$. Then $\vdash_{\omega} C(\mathrm{D}(1-4)) \wedge(\wedge \Phi) \wedge C($ Solv $) \supset A$ for some finite subset $\Phi$ of WD. Hence there is a proof $P$ of $C(\mathrm{D}(1-4)) \wedge(\wedge \Phi) \wedge$ $C$ (Solv) $\supset A$. We substitute $C(\operatorname{Nash}(a))$ for each occurrences of $D_{i}(a)(i=1, \ldots, n$ and $a \in \Sigma)$ in $P$. Then we have a proof $P^{\prime}$ of $C(\mathrm{D}(1-4)[C(\mathrm{Nash})]) \wedge(\wedge \Phi[C(\mathrm{Nash})]) \wedge$ $C$ (Solv) $\supset A$. Note that $C$ (Solv) and $A$ are not affected by these substitutions since they contain no $D_{i}, i=1, \ldots, n$.

Since $C($ Solv $) \vdash_{\omega} C(\mathrm{D}(1-4)[C(\mathrm{Nash})])$ by Proposition 5.1, we have $\vdash_{\omega}(\wedge \Phi[C(\mathrm{Nash})])$ $\wedge C($ Solv $) \supset A$. Also, $C($ Solv $) \vdash_{\omega} \wedge \Phi[C($ Nash $)]$ by using (5.2), since $\Phi$ is a subset of WD. Hence $\vdash_{\omega} C($ Solv $) \supset A$.

Proof of the Second Assertion of (1). Suppose $C(\mathrm{D}(1-4)), \mathrm{WD}, C($ Solv $) \vdash_{\omega} \neg \vee_{x_{x}} D_{i}(x)$. By Theorem 5.A, we have $C(\mathrm{D}(1-4))$,WD, $C($ Solv $) \vdash_{\omega} \neg \vee{ }_{x} C(\operatorname{Nash}(x))$. By Lemma 5.2, we have $C(\operatorname{Solv}) \vdash_{\omega} \neg \vee_{x} C(\operatorname{Nash}(x))$. By Lemma 2.2.(1), we have Solv $\vdash_{0} \neg \vee_{x} \operatorname{Nash}(x)$. However, Solv $\vdash_{0} \vee_{x} \operatorname{Nash}(x)$, which implies that Solv is inconsistent with respect to $\vdash_{0}$. It can be proved that this is not the case.

For the first assertion of Theorem 5.C.(1), we need one metatheorem, which was proved in Kaneko-Nagashima (1997a).

Theorem 5.D. (Disjunctive Property): Let $\Gamma$ be a set of nonepistemic formulae, and $A_{1}, \ldots, A_{k}$ nonepistemic formulae. If $C(\Gamma) \vdash_{\omega} \vee_{t=1}^{k} C\left(A_{t}\right)$, then $C(\Gamma) \vdash_{\omega} C\left(A_{t}\right)$ for some $t=1, \ldots, k$.

Proof of the First Assertion of (1). Suppose $C(\mathrm{D}(1-4)), \mathrm{WD}, C(\mathrm{Solv}) \vdash_{\omega} \vee_{x} D(x)$. Then it follows from Theorem 5.A that $C(\mathrm{D}(1-4)), \mathrm{WD}, C(\mathrm{Solv}) \vdash_{\omega} \vee_{x} C(\mathrm{NaSh}(x))$. Then we have, by Lemma 5.2, $C$ (Solv) $\vdash_{\omega} \vee_{x} C(\operatorname{Nash}(x))$. Applying Theorem 5.D to this statement, we have $C(\operatorname{Solv}) \vdash_{\omega} C(\operatorname{Nash}(a))$ by some strategy profile $a$. By Lemma 2.2.(1), we have Solv $\vdash_{0} \operatorname{Nash}(a)$. Then we construct an assignment based on a game $g$ which has a unique Nash equilibrium different from $a$. Here we need the assumption $\ell_{j} \geq 2$ for at least one $j$. By Soundness for $\mathrm{GL}_{0}^{\mathrm{f}}$, it is not the case that Solv $\vdash_{0} \operatorname{Nash}(a)$.

\section{Unsolvable games}

When $g$ is an unsolvable game, i.e., does not satisfy interchangeability (2.1), Proposition 5.1.(2), a fortiori, Theorem 5.A, fails to hold: $C(\mathrm{Nash}(\cdot))$ does not satisfy Axiom D4. For an unsolvable game, subsolutions play the role of Nash( $\cdot)$, instead, but we will meet two new difficulties. One, purely game theoretical, is that an unsolvable game has multiple subsolutions and the individual choice of a subsolution may lead to a double cross. Therefore they need to share some information on the choice of a subsolution. The other is that, as already discussed in Subsection 2.3, the subsolution concept needs an extensional description. Therefore we need the common knowledge of 
$\mathrm{G}_{g}$ as well as Eq. Once these axioms as well as the choice of a subsolution are assumed to be common knowledge, we would have the characterization result parallel to that obtained in Section 5. Without sharing the knowledge on the choice of a subsolution, however, the game is not playable, again.

\subsection{Exchange of some information to choose a subsolution}

Proposition 3.2 becomes the following form. Here, recall that the equilibrium set $E_{g}$ is divided into the union, $\bigcup_{t=1}^{\sigma} E_{g}^{t}$, of subsolutions.

\section{Proposition 6.1.}

1. Eq, $\mathrm{G}_{g}, \mathrm{D}^{0}(1,2,4) \vdash_{0} \vee_{k=1}^{\sigma} \wedge_{x}\left(D_{i}(x) \supset \operatorname{Sol}^{k}(x)\right)$;

2. $C\left(\mathrm{Eq}, \mathrm{G}_{g}\right), C(\mathrm{D}(1,2,4)) \vdash_{\omega} C\left(\vee_{k=1}^{\sigma} \wedge_{x}\left(D_{i}(x) \supset \mathrm{Sol}^{k}(x)\right)\right)$, where $C\left(\mathrm{Eq}, \mathrm{G}_{g}\right)$ is $C\left(\mathrm{Eq} \cup \mathrm{G}_{g}\right)$.

Proof. (2) follows from (1) and Lemma 2.2.(2). Now we prove (1) in a semantic way.

Let $\tau$ be any truth assignment in which $\mathrm{Eq}, \mathrm{G}_{g}, \mathrm{D}^{0}(1,2,4)$ are true. It follows from Axioms $\mathrm{D} 1^{0}$ and $\mathrm{D} 2^{0}$ that if $D_{i}(a)$ is true, then $a$ is a Nash equilibrium. Hence $\left\{a: \tau\left(D_{i}(a)\right)=\right.$ true $\}$ is a subset of $E_{g}$. Then Axiom D4 implies that the set $\left\{a: \tau\left(D_{i}(a)\right)=\right.$ true\} satisfies interchangeability. Since each subsolution is a maximal set of Nash equilibria satisfying interchangeability, $\left\{a: \tau\left(D_{i}(a)\right)=\right.$ true $\}$ is a subset of some subsolution, say, $E_{g}^{k}$. Thus $\vDash{ }_{\tau} \wedge_{x}\left(D_{i}(x) \supset \operatorname{Sol}^{k}(x)\right)$. This $k$ may depend upon $\tau$, but $\vee_{k=1}^{\sigma}$ $\wedge_{x}\left(D_{i}(x) \supset \operatorname{Sol}^{k}(x)\right)$ is true in any truth assignment $\tau$ satisfying Eq, $\mathrm{G}_{g}, \mathrm{D}^{0}(1,2,4)$. By (2.5), we have Eq, $\mathrm{G}_{g}, \mathrm{D}^{0}(1,2,4) \vdash_{0} \vee_{k=1}^{\sigma} \wedge_{x}\left(D_{i}(x) \supset \mathrm{Sol}^{k}(x)\right)$.

The first states that for a game $g$, if $a$ is a final decision profile, then it belongs to one of the subsolutions, and the second statement is simply a conclusion of the first and Lemma 2.2.(2). In the second, however, which subsolution is implied is unknown, since the disjunction is taken over the subsolutions in the scope of common knowledge. To choose one subsolution, the players need to exchange some information in addition to the common knowledge of $\mathrm{Eq}$ and $\mathrm{G}_{g}$.

We denote the following formula

$$
\wedge_{k \neq t} \underset{x}{\vee}\left(D_{i}(x) \wedge \neg \operatorname{Sol}^{k}(x)\right)
$$

by $\operatorname{Sub}(t)$. This states that for any subsolution other than the $t$-th one, some predicted final decision profile does not belong to the subsolution. If this is common knowledge, the players can choose the subsolution $\mathrm{Sol}^{t}$.

Proposition 6.2. Let $t$ be one of $1, \ldots, \sigma$. Then $C\left(\mathrm{Eq}, \mathrm{G}_{g}\right), C(\mathrm{D}(1-4)), C(\operatorname{Sub}(t)) \vdash_{\omega}$ $\wedge_{x}\left(D_{i}(x) \supset C\left(\operatorname{Sol}^{t}(x)\right)\right)$.

Proof. Since $\vee_{k=1}^{\sigma} \wedge_{x}\left(D_{i}(x) \supset \operatorname{Sol}^{k}(x)\right)$ is equivalent to $\operatorname{Sub}(t) \supset \vee_{x}\left(D_{i}(x) \supset \operatorname{Sol}^{t}(x)\right)$ with respect to $\vdash_{0}$, we have, by Lemma 2.2.(2), $C\left(\mathrm{Eq}, \mathrm{G}_{g}\right), C(\mathrm{D}(1,2,4)) \vdash_{\omega} C[\operatorname{Sub}(t) \supset$ $\left.\wedge_{x}\left(D_{i}(x) \supset \operatorname{Sol}^{t}(x)\right)\right]$. Hence $C\left(\mathrm{Eq}, \mathrm{G}_{g}\right), C(\mathrm{D}(1,2,4)) \vdash_{\omega} C(\mathrm{Sub}(t)) \supset \wedge_{x}\left[C\left(D_{i}(x)\right) \supset\right.$ 
$\left.C\left(\operatorname{Sol}^{t}(x)\right)\right]$ by Lemmas 2.3 and 2.4. Since $C(\mathrm{D}(2,3)) \vdash_{\omega} \wedge_{x}\left(D_{i}(x) \supset C\left(D_{i}(x)\right)\right)$ by Proposition $\quad 3.2 .(1)$, we have $C\left(\mathrm{Eq}, \mathrm{G}_{g}\right), C(\mathrm{D}(1-4)), C(\mathrm{Sub}(t)) \quad \vdash_{\omega} \wedge_{x}\left(D_{i}(x) \supset\right.$ $\left.C\left(\operatorname{Sol}^{t}(x)\right)\right)$.

Thus, the common knowledge of the information, $\operatorname{Sub}(t)$, suffices for $\wedge_{x}\left[D_{i}(x) \supset\right.$ $\left.C\left(\operatorname{Sol}^{t}(x)\right)\right]$. In addition to this, if $C\left(\operatorname{Sol}^{t}(\cdot)\right)$ satisfies $C(\mathrm{D}(1-4))$, then Axiom WD implies that $D_{i}(x)$ is equivalent to $C\left(\operatorname{Sol}^{t}(x)\right)$. The following proposition states that $C\left(\operatorname{Sol}^{t}(\cdot)\right)$ satisfies those axioms under $C\left(\mathrm{Eq}, \mathrm{G}_{g}\right)$, which can be proved in the same manner as in the proof of Proposition 5.1.

Proposition 6.3. $C\left(\mathrm{Eq}, \mathrm{G}_{g}\right) \vdash_{\omega} C\left(\mathrm{D} h\left[C\left(\mathrm{Sol}^{k}\right)\right]\right)$ for $k=1, \ldots, \sigma$ and $h=1,2,3,4$.

By Propositions 6.2 and 6.3 together with Axiom WD, we have following theorem, which is a generalization of Theorem 5.A.

Theorem 6.A. Let $t$ be one of $1, \ldots, \sigma$. Then $C\left(\mathrm{Eq}, \mathrm{G}_{g}\right), C(\mathrm{D}(1-4)), \mathrm{WD}, C(\mathrm{Sub}(t))$ $\vdash_{\omega} \wedge_{x}\left(D_{i}(x) \equiv C\left(\operatorname{Sol}^{t}(x)\right)\right)$.

The role of $C(\operatorname{Sub}(t))$ in Proposition 6.2 is to choose one subsolution. Without this assumption, we could not prove Proposition 6.2, which will be stated as Theorem 6.B. The other direction does not need this assumption.

The assumption, $C(\operatorname{Sub}(t))$, itself is rather an abstract statement, but may be regarded as derived from the information commonly obtained by some communication. In the battle of the Sexes (Table 1), for example, if the players share the knowledge, via talking on the phone (or, face-to-face) that they would choose the pair $\left(\mathbf{s}_{11}, \mathbf{s}_{21}\right)$ as final decisions, then the assumption $C(\operatorname{Sub}(t))$ might be derived.

Since we need to assume $\mathrm{Eq}$ and $\mathrm{G}_{g}$ to describe subsolutions, we already assume the specific knowledge of the structure of the game in Theorem 6.A, contrary to Theorem 5.A. Hence the game $g$ with $\sigma \geq 1$ is playable under the premise of 6.A, i.e.,

$$
C\left(\mathrm{Eq}, \mathrm{G}_{g}\right), C(\mathrm{D}(1-4)), \mathrm{WD}, C(\mathrm{Sub}(t)) \vdash_{\omega} \underset{x}{\vee} D_{i}(x) .
$$

In this case, the problem is the necessity of $C(\operatorname{Sub}(t))$ from the solution-theoretic and playability point of view, which is the subject of the next subsection.

\subsection{Necessity of the exchange of the information}

The next theorem states that without any knowledge of $\mathrm{Sub}(t)$, the choice of one subsolution is impossible.

Theorem 6.B. Suppose that game $g$ has at least two subsolutions, i.e., $\sigma \geq 2$. Then for any $k=1, \ldots, \sigma$ and $i=1, \ldots, n$,

$$
\begin{aligned}
& \text { neither } C\left(\mathrm{Eq}, \mathrm{G}_{g}\right), C(\mathrm{D}(1-4)) \vdash_{\omega}{ }_{x}\left(D_{i}(x) \supset C\left(\mathrm{Sol}^{k}(x)\right)\right) ; \\
& \operatorname{nor} C\left(\mathrm{Eq}, \mathrm{G}_{g}\right), C(\mathrm{D}(1-4)) \vdash_{\omega} \neg \bigwedge_{x}\left(D_{i}(x) \supset C\left(\operatorname{Sol}^{k}(x)\right)\right) .
\end{aligned}
$$


This theorem implies that without sharing some information about a subsolution, a player could not reason which subsolution is played specifically. This implies that (6.2) could not hold without $C(\operatorname{Sub}(t))$, since the premise of WD holds trivially and $D_{i}(a)$ may be always (semantically) false. That is, for any game $g$ with $\sigma \geq 2$,

$$
\begin{aligned}
& \text { neither } C\left(\mathrm{Eq}, \mathrm{G}_{g}\right), C(\mathrm{D}(1-4)), W D \vdash_{\omega} \underset{x}{\vee} D_{i}(x) \\
& \operatorname{nor} C\left(\mathrm{Eq}, \mathrm{G}_{g}\right), C(\mathrm{D}(1-4)), W D \vdash_{\omega} \neg \underset{x}{\vee} D_{i}(x) .
\end{aligned}
$$

Thus, without additional information of which subsolution would be played, the game is, again, not playable.

Proof. Suppose $C\left(\mathrm{Eq}, \mathrm{G}_{g}\right), C(\mathrm{D}(1-4)) \vdash_{\omega} \wedge_{x}\left(D_{i}(x) \supset C\left(\operatorname{Sol}^{k}(x)\right)\right)$ for some $k$. Hence we have, by Lemma 2.2.(1), Eq, $\mathrm{G}_{g}, \mathrm{D}^{0}(1,2,4) \vdash_{\omega} \wedge_{x}\left(D_{i}(x) \supset \mathrm{Sol}^{k}(x)\right)$. Now we show that this is impossible, which implies that the supposition does not hold. For this, we give an assignment $\tau$ on the atomic formulae so that $\mathrm{Eq}, \mathrm{G}_{g}, \mathrm{D}^{0}(1,2,4)$ are true in $\tau$ but $\wedge_{x}\left(D_{i}(x) \supset \operatorname{Sol}^{k}(x)\right)$ is false. This together with Soundness for $\mathrm{GL}_{0}^{\mathrm{f}}$ implies that $\wedge_{x}\left(D_{i}(x) \supset \operatorname{Sol}^{k}(x)\right)$ is not provable from $\left\{\mathrm{Eq}, \mathrm{G}_{g}, \mathrm{D}^{0}(1,2,4)\right\}$ with respect to $\vdash_{0}$.

Let $\operatorname{Sol}^{k^{\prime}}(\cdot)$ be different from $\operatorname{Sol}^{k}(\cdot)$. Then we define a truth assignment $\tau$ as follows:

$$
\begin{aligned}
& (1): \tau\left(\mathbf{s}_{j t}=\mathbf{s}_{j t^{\prime}}\right)= \begin{cases}\text { true } & \text { if } t=t^{\prime} \\
\text { false } & \text { otherwise }\end{cases} \\
& (2): \tau\left(R_{j}(a: b)\right)= \begin{cases}\text { true } & \text { if } g_{j}(a) \geq g_{j}(b) \\
\text { false } & \text { otherwise }\end{cases} \\
& \text { (3): } \tau\left(D_{j}(a)\right)= \begin{cases}\text { true } & \text { if } a \in E_{g}^{k^{\prime}} \\
\text { false } & \text { otherwise }\end{cases}
\end{aligned}
$$

where $j=1, \ldots, n$. Then every axiom of Eq, $\mathrm{G}_{g}, \mathrm{D}^{0}(1,2,4)$ is true, but not $\vDash{ }_{\tau} D_{i}(a) \supset$ $\operatorname{Sol}^{k}(a)$ for some $a$ in $E_{g}^{k^{\prime}}$, since $\tau\left(D_{i}(a)\right)=$ true but not $\vDash{ }_{\tau} \operatorname{Sol}^{k}(a)$ for any $a \in E_{g}^{k^{\prime}}-$ $E_{g}^{k}$. Hence $\wedge_{x}\left(D_{i}(x) \supset \operatorname{Sol}^{k}(x)\right)$ is false in $\tau$.

Next, suppose $C\left(\mathrm{Eq}, \mathrm{G}_{g}\right), C(\mathrm{D}(1-4)) \vdash_{\omega} \neg^{\wedge} \wedge_{x}\left(D_{i}(x) \supset C\left(\mathrm{Sol}^{t}(x)\right)\right)$ for some $k$. By Proposition 2.2.(1), we have Eq, $\mathrm{G}_{g}, \mathrm{D}^{0}(1,2,4) \vdash_{0} \neg \wedge_{x}\left(D_{i}(x) \supset \operatorname{Sol}^{t}(x)\right)$. Equivalently, $\mathrm{Eq}, \mathrm{G}_{g}, \mathrm{D}^{0}(1,2,4) \vdash_{0} \vee_{x}\left(D_{i}(x) \wedge \neg \operatorname{Sol}^{k}(x)\right)$. Let $b \in E_{g}^{k^{\prime}}-E_{g}^{k}$. We modify the above truth assignment $\tau$ by changing (3) into

$$
\left(3^{\prime}\right): \tau\left(D_{j}(a)\right)=\text { false for all } a \text { and } j=1, \ldots, n \text {. }
$$

In the truth assignment $\tau$ defined, $\vee_{x}\left(D_{i}(x) \wedge \neg \operatorname{Sol}^{k}(x)\right)$ is false but $\mathrm{D}^{0}(1,2,4)$ is true in the trivial way. Hence this is a contradiction.

To choose one subsolution, the players need to communicate with each other to share some information. The following theorem states that the sufficient condition given in the previous subsection is the deductively weakest, whose proof is omitted. 
Table 5

\begin{tabular}{llll}
\hline & $\mathbf{s}_{21}$ & $\mathbf{s}_{22}$ & $\mathbf{s}_{23}$ \\
\hline $\mathbf{s}_{11}$ & $(0,0)$ & $(1,1)^{*}$ & $(1,1)^{*}$ \\
$\mathbf{s}_{12}$ & $(1,1)^{*}$ & $(1,1)^{*}$ & $(0,0)$ \\
$\mathbf{s}_{13}$ & $(1,1)^{*}$ & $(0,0)$ & $(1,1)^{*}$ \\
\hline
\end{tabular}

Theorem 6.C. Let $t$ be one of $1, \ldots, \sigma$, and $\Gamma$ any set of formulae. If $C\left(\mathrm{Eq}, \mathrm{G}_{g}\right), C(\mathrm{D}(1-$ $4)), \Gamma \vdash_{\omega} C\left(\wedge_{x}\left(D_{i}(x) \equiv C\left(\operatorname{Sol}^{t}(x)\right)\right)\right)$, then $C\left(\mathrm{Eq}_{,} \mathrm{G}_{g}\right), C(\mathrm{D}(1-4)), \Gamma \vdash_{\omega} C(\operatorname{Sub}(t))$.

The condition $C(\operatorname{Sub}(t))$ eliminates all the possibilities but the $t$-th subsolution. However, more specific information may suffice. For example, the following is one possible assumption on the game $g$,

(Dsub): $E_{g}^{1}, \ldots, E_{g}^{\sigma}$ are mutually exclusive.

The battle of the sexes (Table 1) satisfies this condition. For a game satisfying Dsub, an exchange of an indication of a final decision profile suffices, instead of the elimination of possibilities. That is, for a game satisfying Dsub, the sufficient condition, $C(\operatorname{Sub}(t))$, can be replaced by $C\left(\vee_{x}\left(D_{i}(x) \wedge \operatorname{Sol}^{t}(x)\right)\right)$. This can be proved in the same way as Theorem 6.A. In the game of Table 2 , Dsub is not sufficient, since $\vee_{x}\left(D_{i}(x) \wedge \operatorname{Sol}^{1}(x)\right)$ does not eliminate the possibility $C\left(\vee_{x}\left(D_{i}(x) \wedge \operatorname{Sol}^{2}(x)\right)\right)$. Nevertheless, it suffices to exchange the information of some particular pair, for example, the common knowledge that $\left(\mathbf{s}_{12}, \mathbf{s}_{21}\right)$ is a final decision pair is sufficient, that is,

$$
C\left(\mathrm{Eq}, \mathrm{G}_{g}\right), C(\mathrm{D}(1-4)), \mathrm{WD}, C\left(D_{i}\left(\mathbf{s}_{12}, \mathbf{s}_{21}\right)\right) \vdash_{\omega} C\left({ }_{x}\left(D_{i}(x) \equiv C\left(\operatorname{Sol}^{1}(x)\right)\right)\right) .
$$

On the other hand, the exchange of the information of $\left(\mathbf{s}_{11}, \mathbf{s}_{21}\right)$ is not sufficient.

The game in Table 5 has six subsolutions and each Nash equilibrium belongs to exactly two subsolutions. In this game, the exchange of the information of choosing one subsolution needs to have the original form, $C(\operatorname{Sub}(t))$.

\section{Remarks on the final decision axioms}

\subsection{Role of each axiom}

Consider the role of each of Axioms D1-D4 by eliminating it from the others. The elimination of D1 or D2 makes our theory almost meaningless, and without Axiom D3 or D4, the theory would still have some structure but become much poorer.

(1) Axiom D1: Without D1, we would have the same infinite regress. The deductively weakest formula satisfying $C(\mathrm{D}(2,3,4))$ is given as the common knowledge of a strategy profile under the assumption $C\left(\mathrm{Eq}, \mathrm{G}_{g}\right)$, i.e.,

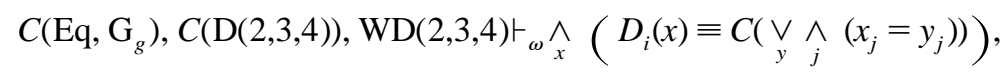


where $\mathrm{WD}(2,3,4)$ is the modification of WD by eliminating $\mathrm{D} 1$. This states that the prediction is the common knowledge of a strategy profile. Thus, the trivial structure is given by these axioms.

(2) Axiom D2: Without D2, we would not meet the common knowledge of the other axioms. Each player has $D_{i}(\cdot)$ but does not think about the other players' predictions. Hence the others' choices remain arbitrary for him. That is, we have

$$
\mathrm{D}(1,3,4), \mathrm{WD}^{-}(1,3,4) \vdash_{\omega} \wedge_{x}\left(D_{i}(x) \equiv K_{i}\left(\widehat{\wedge}_{y_{i}} R_{i}\left(x: y_{i}, x_{-i}\right)\right)\right) \quad \text { for } i=1, \ldots, n .
$$

Here $\mathrm{WD}^{-}(1,3,4)$ does not require the common knowledge operator for its premise. Thus, there are $n$ independent decision problems without interactions, though the situation itself may be interactive.

(3) Axiom D3: We already discussed the case without D3 in Subsection 5.2.

(4) Axiom D4: Without D4, our theory would be simpler. The infinite regress discussed in Section 5 remains unchanged. The main theorem becomes

$$
C(\mathrm{D}(1,2,3)), \mathrm{WD}(1,2,3) \vdash_{\omega} \wedge_{x}\left(D_{i}(x) \equiv C(\operatorname{Nash}(x))\right) .
$$

This does not need the interchangeability condition. In this case, we would loose the demarcation between the solvable and unsolvable games. Thus, the entire consideration of Section 6 would disappear. The role of D4 could be found by looking at a different formulation of our axiomatization, which is given in the next subsection.

\subsection{The I-system of final decision axioms}

We presented the axioms on final decisions $D_{1}(\cdot), \ldots, D_{n}(\cdot)$ as $n$-ary predicates. That is, a prediction is an attribute of a final decision profile. Therefore each player $i$ makes simultaneously a prediction of a strategy profile for all the players. Kaneko-Nagashima (1991, 1996) and Kaneko (1997b) adopted different axiomatic systems so that predictions are separately made for each player. Player $i$ makes a prediction of player $j$ 's choice, i.e., it is an attribute of a strategy for each player instead of a strategy profile. We call this system the I-system, and the system of the present paper the D-system.

To formulate the I-system, we prepare unary predicates $I_{11}(\cdot), \ldots, I_{1 n}(\cdot) ; \ldots ; I_{n 1}(\cdot), \ldots$, $I_{n n}(\cdot)$ and assume the following base axioms: for each $i=1, \ldots, n$,

$\operatorname{Axiom~} \mathbf{I 1}_{i}^{0} \cdot \wedge_{x}\left(\wedge_{j} I_{i j}\left(x_{j}\right) \supset \wedge_{y_{i}} R_{i}\left(x_{i} ; x_{-i}: y_{i} ; x_{-i}\right)\right)$

$\operatorname{Axiom~} \mathbf{I}_{i}^{0} \cdot \wedge_{x} \wedge_{j} \wedge_{k}\left(I_{i j}\left(x_{j}\right) \supset I_{k j}\left(x_{j}\right)\right)$

$\operatorname{Axiom} \mathbf{I} 3_{i}^{0} \cdot \wedge_{x} \wedge_{j}\left(I_{i j}(x) \supset K_{i}\left(I_{i j}(x)\right)\right)$;

Axiom $\mathbf{I 4}_{i}^{0} \cdot \wedge_{i} \wedge_{j} \wedge_{k}\left(\vee_{x_{i}} I_{i j}\left(x_{j}\right) \supset \vee_{x_{j}} I_{i k}\left(x_{k}\right)\right)$

We define $\mathrm{I} 1_{i}, \ldots, \mathrm{I} 4_{i}$ to be $K_{i}\left(\mathrm{I} 1_{i}^{0}\right), \ldots, K_{i}\left(\mathrm{I} 4_{i}^{0}\right)$. Axioms D4 and $\mathrm{I} 4$ connect the D-system and I-system in the following sense: 


$$
\vdash_{\omega} I 4_{i}^{0} \wedge\left({ }_{x}^{\wedge}\left[D_{i}(x) \equiv \wedge_{j} I_{i j}\left(x_{j}\right)\right]\right) \equiv D 4_{i}^{0} \wedge\left(\wedge_{j} \wedge_{x_{j}}\left[I_{i j}\left(x_{j}\right) \equiv \underset{y_{-j}}{\vee} D_{i}\left(x_{j}, y_{-j}\right)\right]\right) .
$$

Thus, we can start either with the I-system together the definition of $D_{i}(a)$ to be $\wedge_{j} I_{i j}(a)$ or either with the D-system together the definition of $I_{i j}\left(a_{j}\right)$ to be $\vee_{y_{-j}} D_{i}\left(a_{j}, y_{-j}\right)$. Furthermore, we can prove the entire equivalence between the above two systems with the above connection.

\subsection{Fully and partially interactive games}

Ignoring the difference between the D-system and I-system, the axiomatization in this paper is a special case of that in Kaneko (1997b). In his axiomatization, multiple systems of axioms for decision making may be permitted, depending upon a game. Kaneko (1997b) gave a condition on a game only for the system of this paper to be permissible. A game is called fully interactive iff it satisfies this condition, and otherwise, it is called (properly) partially interactive. The system in this paper is permitted for any games, though partially interactive games may have multiple "weaker" systems of axioms.

Here we give some simple examples of fully and partially interactive games. In the Prisoner's Dilemma (Table 3), since each player has a dominant strategy, $\mathbf{s}_{i 2}$, it is possible for each player $i$ to make a decision satisfying utility maximization only with the knowledge of his own payoff function but without predicting the other's decision. This argument is formulated as one system of axioms in (Kaneko, 1997b), but the solution for this system does not require the knowledge of the other player's decision. This argument is not applied to the game of Table 4, since only player 1 has a dominant strategy, $\mathbf{s}_{12}$. In this game, 1 can ignore 2's choice, but 2 needs to predict 1's choice. This situation is also formulated as a system of axioms. Even in these games, if we require each player to infer the other's decision, we would have the system in this paper.

The game of Table 6 obtained by adding one strategy to player 2 to Table 4 is fully interactive: this has the same Nash equilibrium $\left(\mathbf{s}_{12}, \mathbf{s}_{22}\right)$, but without predicting the other's decision, each player cannot make a decision to maximize his payoff. This game differs from the games of Tables 3 and 4 in that the system in this paper is only permissible.

In fact, there is a great spectrum of games from those with dominant strategies to fully interactive games. One typical (four-person) example is as follows: each of 1,3 and each of 2,4 have three and two strategies, respectively. The payoffs for players 1 and 2 are determined by their own strategies, which are given by Table 6 . Those for 3 and 4 depend upon their strategies as well as the choices of 1 and 2: if 1 and 2 choose $\left(\mathbf{s}_{12}, \mathbf{s}_{22}\right)$, then the payoffs of 3 and 4 are given also as Table 6 with the replacements of players

Table 6

3, 4's payoffs if $\left(\mathbf{s}_{12}, \mathbf{s}_{22}\right)$ is played

\begin{tabular}{llll}
\hline & $\mathbf{s}_{21}$ & $\mathbf{s}_{22}$ & $\mathbf{s}_{23}$ \\
\hline $\mathbf{s}_{11}$ & $(5,5)$ & $(1,2)$ & $(3,3)$ \\
$\mathbf{s}_{12}$ & $(6,1)$ & $(3,3)^{*}$ & $(0,2)$ \\
\hline
\end{tabular}


Table 7

Otherwise

\begin{tabular}{llll}
\hline & $\mathbf{s}_{41}$ & $\mathbf{s}_{42}$ & $\mathbf{s}_{43}$ \\
\hline $\mathbf{s}_{31}$ & $(3,3)$ & $(6,1)$ & $(0,2)$ \\
$\mathbf{s}_{32}$ & $(1,6)$ & $(5,5)$ & $(3,3)$ \\
\hline
\end{tabular}

1,2 with 3,4; and if 1 and 2 choose a strategy pair other than $\left(\mathbf{s}_{12}, \mathbf{s}_{22}\right)$, then their payoff matrix is given by Table 7 (obtained from 6 by permuting the roles of $\mathbf{s}_{i 1}$ and $\mathbf{s}_{i 2}$ for $i=3,4)$. In this game, if 1 and 2 ignore 3 and 4 , then 1 and 2 are facing the game of Table 6, which is fully interactive. Players 3 and 4 still need to infer the choices of 1,2, and their part is fully also regarded as fully interactive.

When a partially interactive game permits a "weaker" system of axioms, it has a part which can be regarded as fully interactive in that each player needs to predict the others' decisions in the part, though sometimes it is trivial in the sense that it consists of a single player. The system of axioms restricted to such a fully interactive part of the game is regarded as the same as the system in this paper. Therefore our analysis is applied also to the fully interactive parts of partially interactive games. See Kaneko (1997b).

In the approach of this paper, we used the game logic $\mathrm{GL}_{\omega}$ where the knowledge of the logical and introspection abilities are allowed up to any finite depths. Kaneko's (1997b) axiomatization permits, for some games, to be discussed in game logic $\mathrm{GL}_{m}$ for a finite $m$. For example, the Prisoner's Dilemma can be discussed in logic $\mathrm{GL}_{1}$, where each player does not think about the other player's thinking. Nevertheless, when we consider the axiomatic system of this paper, we need the limit logic $\mathrm{GL}_{\omega}$, and this is inevitable for many games.

\subsection{Comparisons with Johansen's argument}

Now we look at Johansen's (1982) argument on Nash equilibrium from the viewpoint of the above axiomatization. Johansen proposed four postulates for rational (individualistic) decision making in a game. We reproduce them with slight modifications in terminology:

(J1). A player makes his decision $a_{i} \in \Sigma_{i}$ on the basis of, and only on the basis of information concerning the strategy sets $\Sigma_{1}, \ldots, \Sigma_{n}$, and preferences $R_{1}, \ldots, R_{n}$.

(J2). In choosing his own decision, a player assumes that the other players are rational in the same way that he himself is rational.

(J3). If some decision is the rational decision to make for an individual player, then this decision can be correctly predicted by the other players.

(J4). Being able to predict the actions to be taken by the other players, a player's own decision maximizes his preference relation corresponding to the predicted actions of the other players. 
Johansen argues that if a game possesses a unique Nash equilibrium, then each player's Nash strategy is the unique choice satisfying these four postulates. He claims also that the uniqueness of a Nash equilibrium is for simplicity and that interchangeability suffices for his argument (Johansen (1982), p.424). Since Johansen did not formalize his postulates and arguments at our level, a lot of ambiguities remain - Bernheim (1986), p.486 criticized Johansen's postulates as ambiguous and self-referential. Nevertheless, it might be useful for the understanding of our and his approaches to make comparisons of them.

Besides the differences in formalisms, there is one difference we should be conscious of. Johansen's postulates describe the whole situation he is considering. On the other hand, our axioms constitute a part of the description of the game situation - the description of logical abilities is made separately in $\mathrm{GL}_{\omega}$. Therefore we should consider the correspondence between his postulates and our entire approach. Postulates J1 and J4 are clear enough even in the comparisons with our approach. We consider the possible interpretations of $\mathrm{J} 2$ and $\mathrm{J} 3$.

Postulate $\mathrm{J} 2$ may be interpreted in our axiomatization as meaning that each player $i$ follows the final decision axioms assuming that the other players also assume the same axioms. This interpretation may be formulated as assuming $K_{i}(\mathrm{D}(1-4))$ for $i=1, \ldots, n$. As remarked above, it is interpreted also as including the assumption that every player has a (complete) logical ability as well as knows (or assumes) that the other players have the same logical abilities. In the latter sense, J2 partially corresponds to our $\mathrm{GL}_{\omega}$ (which may be an overinterpretation of Johansen).

Postulate J3 corresponds to Lemma 3.3 (thus, D2 and D3 combined) rather than D3 itself. This postulate requires each player to assume that the others obey the same postulates as his. Thus, the four postulates require the players to know the postulates themselves for their "rational decision making". In this sense, Johansen's argument looks self-referential, but this self-referential is too ambiguous to be investigated. In our case, the ambiguity is avoided by formulating the whole situation in game logic, and the self-referential becomes the infinite regress, which was solved in Sections 5 and 6.

Johansen's postulates appear to have no counterpart of Axiom D4 ${ }_{i}^{0}$, though Johansen himself emphasized independent individual decision making. Probably, he presupposed a game with a unique Nash equilibrium and needed no explicit postulate for independent decision making.

\section{Acknowledgements}

The research of this paper was partially supported by the Tokyo Center of Economic Research. The author thanks P. Mongin and a referee for valuable comments on an earlier version of the paper.

\section{References}

Aumann, R.J., Brandenberger, A., 1995. Epistemic conditions for Nash equilibrium. Econometrica 63, 1161-1180. 
Bacharach, M., 1987. A theory of rational decision in games. Erkenntnis 27, 17-55.

Bernheim, B.D., 1986. Axiomatic characterizations of rational choice in strategic environments. Scandanavian Journal of Economics 88, 473-488.

Halpern, J.H., Moses, Y., 1992. A guide to completeness and complexity for modal logics of knowledge and beliefs. Artificial Intelligence 54, 319-379.

Jansen, M.J.M., 1981. Maximal Nash subsets for bimatrix game. Naval Research Logistics Quarterly 28, $147-152$.

Johansen, L., 1982. On the status of the Nash type of noncooperative equilibrium in economic theory. Scandanavian Journal of Economics 84, 421-441.

Kaneko, M., Nagashima, T., 1991. Final decisions, Nash equilibrium and solvability in games with the common knowledge of logical abilities. Mathematical Social Sciences 22, 229-255.

Kaneko, M., Nagashima, T., 1996. Game logic and its applications I. Studia Logica 57, 325-354.

Kaneko, M., Nagashima, T., 1997a. Game logic and its applications II. Studia Logica 58, 273-303.

Kaneko, M., Nagashima, T., 1997b. Axiomatic indefinability of common knowledge. In: Bacharach, M., Gerard-Varet, L.A., Mongin, P., Shin, H. (Eds.), Finitary Logics, Epistemic Logic and the Theory of Games and Decision, Kluwer Academic, pp. 69-93.

Kaneko, M., 1998. Extensions of the Depth Lemma in Game Logic. To be completed.

Kaneko, M., 1996. Common Knowledge Logic and Game Logic, ISEP.DP.694, August. To appear in Journal of Symbolic Logic.

Kaneko, M., 1997. Mere and Specific Knowledge of the Existence of a Nash Equilibrium, IPPS. DP. 741.

Kaneko, M., 1997. Decision Making in Partially Interactive Games I: Game Theoretical Development, IPPS. DP. 743. Part II will be completed.

Kats, A., Thisse, J.-F., 1992. Unilaterally competitive games. International Journal of Game Theory 21, 291-299.

Lismont, L., Mongin, P., 1994. On the logic of common belief and common knowledge. Theory and Decision 37, 75-106.

Nash, J.F., 1951. Noncooperative games. Annals of Mathematics 54, 286-295.

Ohnishi, M., Matsumoto, K., 1957. Gentzen method in modal calculi I. Osaka Mathematical Journal 9, $113-130$. 